IA WRE NCE WWEAMCAE WATIONAL LAESAMTOAY.

Lattice Boltzmann

Simulation of Particle Laden Flows in Microfluidic Systems

Dr. D. S. Clague, T. Weisgraber, E. Wheeler, P. Krulevitch, G. Hon, J. Radford, P. Gascoyne, R. Smity, D. Liepmann, C. Meinhart, J. Santiago

July 22, 2003 
This document was prepared as an account of work sponsored by an agency of the United States Government. Neither the United States Government nor the University of California nor any of their employees, makes any warranty, express or implied, or assumes any legal liability or responsibility for the accuracy, completeness, or usefulness of any information, apparatus, product, or process disclosed, or represents that its use would not infringe privately owned rights. Reference herein to any specific commercial product, process, or service by trade name, trademark, manufacturer, or otherwise, does not necessarily constitute or imply its endorsement, recommendation, or favoring by the United States Government or the University of California. The views and opinions of authors expressed herein do not necessarily state or reflect those of the United States Government or the University of California, and shall not be used for advertising or product endorsement purposes. 


\title{
Lattice Boltzmann Simulation of Particle Laden Flows in Microfluidic Systems
}

\author{
By Dr. D. S. Clague \\ LLNL, Center for Micro and Nanotechnology
}

LLNL Team:

Dr. Todd Weisgraber, (3-6349)

Dr. Elizabeth Wheeler (4-6245)

Mr. Gary Hon (Continuing student at UCB)

Mr. Jaret Radford (Continuing student at MIT)

\section{Collaborators:}

Dr. Peter Gascoyne (MD Anderson Cancer Center)

Dr. Rosemary Smity (UC Davis)

Dr. Dorian Liepmann (UC Berkeley)

Dr. Carl Meinhart (UCSB), had interactions with

Dr. J. Santiago (Stanford), had interactions with

Dr. Peter Krulevitch (LLNL), had interactions with

\begin{abstract}
The goal of this effort was to develop dynamic simulation tools to study and characterize particulate transport in Microfluidic devices. This includes the effects of external fields and nearfield particle-particle, particle-surface interactions. The unique aspect of this effort is that we focused on the particles in suspension and rigorously accounted for all of the interactions that they experienced in solution. In contrast, other numerical methods within the program, finite element and finite volume approaches, typically treat the suspended species as non-interacting point particles. Later in the program, some of these approaches incorporated approximations to begin to account for particle-particle interactions.

Through the programs (BioFlips and SIMBIOSYS), we developed collaborative relationships with device-oriented efforts. More specifically and at the request of the SIMBIOSYS program manager, we allowed our efforts/milestones to be more guided by the needs of our BioFlips colleagues; therefore, our efforts were focused on the needs of the MD Anderson Cancer Center (Peter Gascoyne), UCDavis (Rosemary Smith), and UC Berkeley (Dorian Liepmann). The first two collaborations involved the development of Dielectrophoresis analysis tools and the later involved the development of suspension and fluid modeling tools for microneedles.
\end{abstract}

\section{Table of Contents}

Lattice Boltzmann Simulation of Particle Laden Flows in Microfluidic Systems ...................... 1

LLNL Team: 


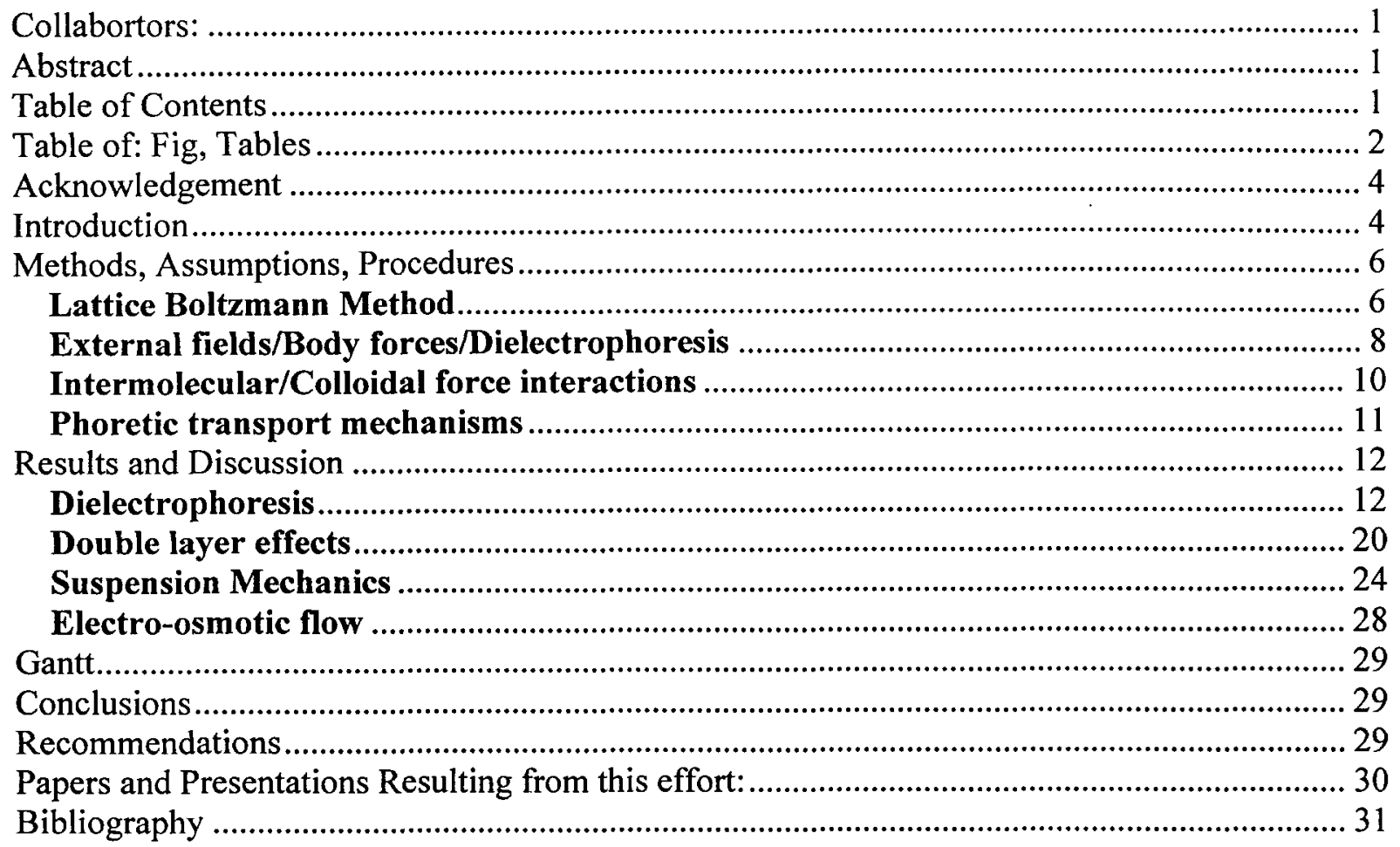

\section{Table of Figures,}

Figure 1. The original road map for proposed effort. The light blue boxes represented near term milestones and the dark blue boxes represented longer term milestones. .............................. 4

Figure 2 A basic block diagram showing the architecture of the module LB capability. The force filter, where time and length scales are converted from real space to lattice space and from lattice space to real space, is central to the overall capability.

Figure 3 Left panel: Interdigitated gold electrodes. The flow is from left to right. The e-field strength is at a maximum at the electrode edges. The right panel shows the e-field strength at the edge of the electrodes as a function of applied voltage.

Figure 4 Typical Dielectrophoretic separator. The dashed rectangular region represents the simulation cell. The simulation cell moves with the target species in the flow direction toward the interdigitated electrode array, denoted by gold rectangles.

Figure 5 Interdigitated electrode configuration and the electric field intensity produced by this electrode configuration. The gold rectangles in the right panel represent electrodes in the array. The surface plot of electric field intensity emanating from the electrode surface...... 13

Figure 6 Gradients in the electric field intensity. The left pannel is the gradient in the flow direction and the right panel is the gradient in the vertical direction.................................. 13

Figure 7 The dependence of the magnitude of the gradient in the electric field strength. The left panel is the dependence on the applied voltage. The right panel is the dependence on the spacing between electrodes.

Figure 8 Determination of the critical Renolds number for a given electrode configuration, applied voltage and particle radius. The left panel shows particle translation as a function of 
Reynolds number relative to an electrode, denoted in gold. The right panel is a phase diagram for predicting particle trapping given a Clausius-Mossotti factor and a channel Reynolds number.

Figure 9 Critical Reynolds number for particle trapping as a function of applied voltage (left panel) and as a function of target species radius, a (right panel).

Figure 10 Dielectrophoretic particle trapping as a function of particle height for a given applied voltage, electrode configuration, Clausius-Mossoti factor and species radius.

Figure 11 Dielectrophoretic response of white blood cells. The goal is to determine the cross over frequencies to optimize the Dielectrophoretic separation protocol.

Figure 12 Multispecies simulation capability. Species in solution can be attributed with unique physical properties, e.g., Dielectrophoretic properties.

Figure 13 Traveling wave Dielectrophoretic separator. Antibody analyte aggregates translating independent of the fluid to a detection region.

Figure 14 Traveling wave Dielectrophoresis translate the target species independent of the fluid (top). The driving force is the gradient in the phase of the electric field (right panel)........ 18

Figure 15 Translocation velocity of target species predicted from the balance of the traveling wave force and the viscous force exerted by the fluid on the body. The right panel is a map of the resulting traveling wave velocities as a function of position from the electrode array.

Figure 16 Predicted translocation velocities as a function of particle size and applied voltage... 19

Figure 17 The details of the electric double layer incorporated into a respesentative particle with the appropriate effective conductivity, $\sigma$, and effective dielectric, $\varepsilon$, properties............... 20

Figure 18 Polystyrene bead. Physical properties taken from Hughes et al., 1999......................................... 21

Figure 19 The real part of the frequency dependent Clausius-Mossotti factor for Polystyrene as a

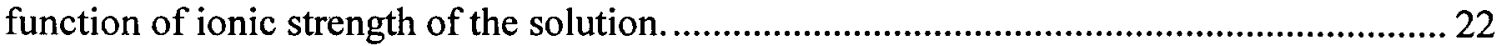

Figure 20 Typical properties of proteins............................................................................. 22

Figure 21 The real part of the Clausius-Mossotti factor for a typical protein at two different fluid

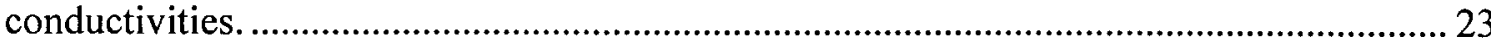

Figure 22 Liposomes with a manufactured biolayer........................................................... 23

Figure 23 The real part of the Clausius-Mossotti factor for a typical protein at two different fluid conductivities.

Figure 24 Experimental results of particle coagulation through a sudden contraction (Results curtesy of Professor Dorian Liepmann).

Figure 25 Lattice Boltzmann representation of sudden contraction in a microneedle. The left panel represents the velocity field and the right panel is the magnitude of the pressure field.

Figure 26 Lattice Boltzmann capability to handle concentrated suspensions. The left panel is the suspension initialization at a specific concentration. The right panel is a snap-shot of particle positions in a rectangular channel in pressure driven flow.

Figure 27 Suspension velocity profile compared with a pure fluid velocity profile (left panel), and the collision frequency, e.g., suspension temperature, on the right.

Figure 28 The velocity profile produced by Electro-Osmotic boundary condisions in a rectangular channel. The the boundary condition at the channel wall was set by the Smolucalski equation for thin double layers.

Figure 29 Gantt Chart for the project aims as put forward in the original propsal. 


\section{Acknowledgement}

The authors would like to acknowledge LLNL/LDRD (Center for Computational Engineering) and DARPA (Bio-flips Program) for financially supporting this effort. Furthermore, we would like to acknowledge the Center for Microtechnology for providing experimental insight into the observed phenomena. This work was performed under the auspices of the U.S. Department of Energy by University of California Lawrence Livermore National Laboratory under contract No. W-7405-Eng-48.

\section{Introduction}

This project was originally a two-year program to develop unique microfluidic modeling software with a specific focus on the suspended species in solution and the unique near-field interactions that occur in microenvironments, more specifically in microfluidic systems. In addition this effort focused on the inclusion of external fields and in particular AC- electrokinetic effects. A flow diagram or road map describing the major goals of the effort is given below in Figure 1 .

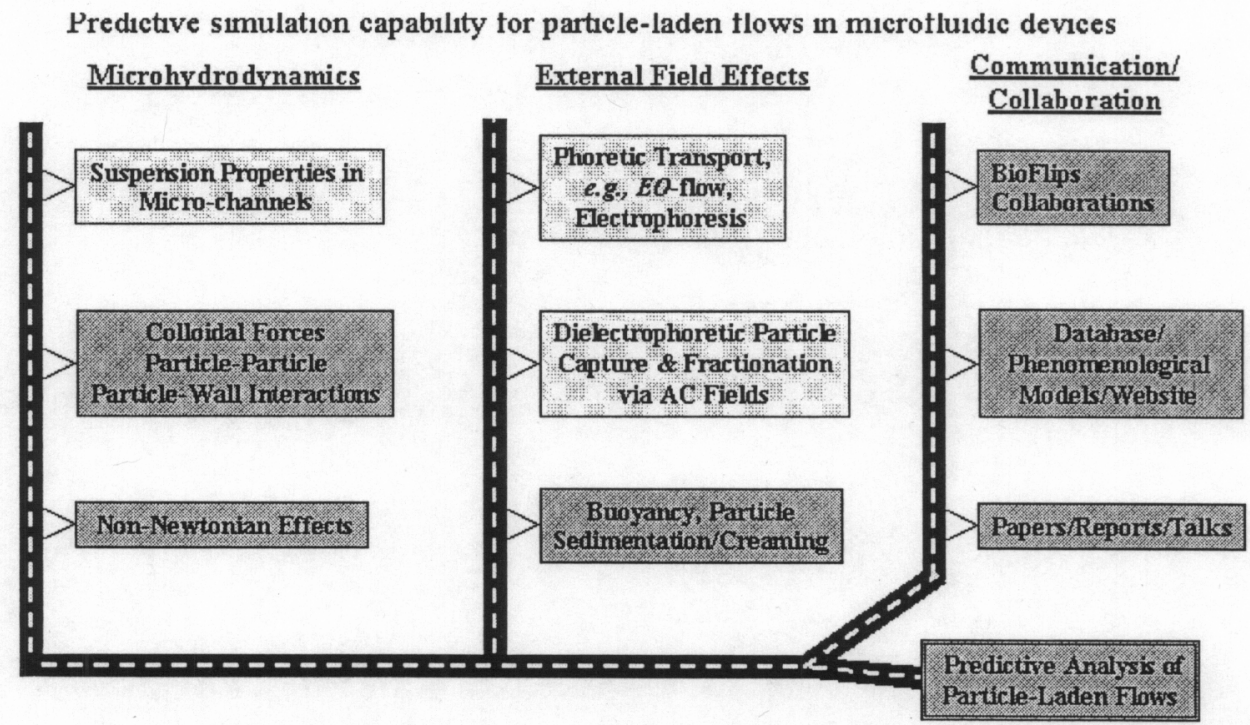

Figure 1. The original road map for proposed effort. The light blue boxes represented near term milestones and the dark blue boxes represented longer term milestones.

This road map was more than merely describing the proposed effort; In a greater sense, it was proposed to and shown to give a larger picture to the needs of modeling in microfluidics. To develop these unique computational capabilities, we developed 3D, parallel lattice Boltzmann simulation capabilities [1].

The Lattice Boltzmann (LB) is uniquely suited for studying the dynamic behavior of macromolecules in microfluidic devices. Current methods - Finite Element Analysis (FEA) and Boundary Element Methods (BEM) - have been useful for modeling and simulating pure fluid flows in microfluidic networks with coupled heat transfer and limited electrokinetic phenomena. 
The current microfluidics simulation tools do not, however, explicitly take into account the behavior of the suspended phase (macromolecules). While valuable, these approaches are limited in crucial ways; namely, it is very difficult to include finite solutes with complex geometries in FEA methods. BEM can easily incorporate mobile particles with external field effects; however, this approach is only valid for Newtonian flows in either the zero or infinite Reynolds number flow regimes. As a consequence, it is not possible to study non-Newtonian effects or small but finite Reynolds number, e.g., $0<\operatorname{Re}<10$, flow effects that can occur in microfluidic devices. The lattice Boltzmann method does not suffer from these limitations. The strength of the LB method is that it is extremely flexible. More specifically, it is very easy to incorporate complex boundaries such as curved bounding walls, obstacles and mobile solutes with complex shapes. The only limitation is the user's ability to mathematically describe the geometry. The LB method easily permits the inclusion of new physics. The capability permits the inclusion of new physics, such as, external $\mathrm{AC}$ and DC field effects (dielectrophoresis and electrokinetic phenomena) and colloidal interactions (electrostatic and van der Waal interactions) that act between suspended solutes and between solutes and substrates, in the LB method. Furthermore, the governing equations used in the LB method naturally take into account both viscous and inertial fluid effects; consequently, the LB method can be used to study finite Reynolds number flows. Additionally, the viscous term can be modified to take into account non-Newtonian fluid effects. To date, microfluidic simulation capabilities deal only with the suspending fluid. The critical need is for a simulation capability that can accurately predict the dynamic behavior of the particles in particle-laden flows while properly accounting for all of the relevant physics encountered in microfluidic systems.

During the program, we developed an enhanced, modular lattice Boltzmann (LB) simulation capability to predict the dynamic transport properties of particle-laden flows in microfluidic sub-systems. The specific physics capability that we developed included dielectrophoresis (DEP), buoyancy, double layer effects and electrokinetic phenomena. These foci where driven by customer needs within the BioFlips program. Through the program we developed dynamic simulation capabilities to study and characterize DEP forces, DEP trapping, Traveling wave, $t w D E P$, DEP force estimation capabilities, new methodologies to predict double layer effects on DEP forces, and fluid and suspension effects in microchannels and sudden contractions. The capabilities developed enable the characterization of multi-species DEP separations, and multi-species suspension behavior in microenvironments. The capabilities are 3D and parallel. Additionally, the particles or suspended species in solution are fully coupled, that is to say, the particles interact with each other and with their environment without approximation. In contrast, most other methods, including other methods supported within the program, have to use an approximation to take into account particle-particle and particleenvironment interactions.

As mentioned, our effort was focused on the overlap between the needs of our customers within the program and our proposed goals. Collaborations were formed with predominantly two Bioflips teams, that is, MD Anderson Cancer Center (Dr. Peter Gascoyne) and UC Davis ( Professor Rosemary Smith). In addition to these collaborations, we also performed collaborative research with Professor Dorian Liepmann (UC Berkelely) and had some interactions with Professors Carl Meinhart and Juan Santiago. Through the program, we are still supporting Professor Rosemary Smith's BioFlips efforts today, and we have now co-authored two new proposals with Professor Gascoyne. Also as a result of the program, we have hired one of Professor Liepmann's former students. Additionally, Professor Meinhart and I have met several 
times to discuss research, and I have now sponsored a student of his to work with me at LLNL twice. I have also visited Professor Santiago and given an invited talk at Stanford. I continue to support and mentor a student of his that works at LLNL.

The lattice Boltzmann capability is applicable to a wide variety of phenomena relevant to microfluidics and other fields that involve complex interactions in particle-laden flows. The proposed capability is revolutionary in that it is focused on the individual and collective behavior of macromolecules for particle-laden flow in microfluidic devices. Because the relevant physics involved will be accounted for, the proposed capability will be useful in revealing new physical phenomena that could in turn could lead to new microfluidic technologies. Furthermore, this capability constitutes a general framework where the user can include field and force effects of choice, e.g., trapping of magnetic beads via magnetic fields, acoustic focusing of macromolecules, electro-osmotic effects, electrophoretic and magnetophoretic separations to name a few. Beyond the microfluidics community, it has wide application and will enable the study of colloidal/macromolecular transport in physiological systems, such as, blood filtration in the kidney, contaminant transport in soils, and it will contribute to understanding new physics associated with nanoparticle behavior in support of the Nanotechnology initiative. This revolutionary dynamic simulation capability will provide device designers a valuable tool to augment the device design process and to explore new sub-system design concepts via computer simulation. The proposed simulation capability will include the necessary physics modules to accurately account for external field effects, intermolecular force interactions, and phoretic transport effects on individual and collections of macromolecules in microchannels (particle laden flows).

\section{Methods, Assumptions, Procedures}

\section{Lattice Boltzmann Method}

The LB method is a mesoscopic description of fluid behavior. In this approach, one does not track individual fluid molecules but tracks a probability distribution function that represents the collective behavior of fluid molecules in local regions. The governing equation solved in the lattice Boltzmann method, i.e., the lattice Boltzmann equation (LBE), is a discrete form of the Boltzmann transport equation [1]. The following equation is the LBE in the simplest form:

$$
f_{i}(\underline{x}+\Delta \underline{x}, t+\Delta t)=f_{i}(\underline{x}, t)+\frac{f_{i}(\underline{x}, t)-f_{i}^{e q}(\underline{x}, t)}{\tau} .
$$

$f_{i}$ is the $i^{\text {th }}$ component of the single-particle-velocity-distribution function, $\tau$ is the scalar relaxation time, which is directly related to the kinematics viscosity, $v$, of the fluid, i.e., $v=(2 \tau-1) / 6$, and $f_{i}^{\text {eq }}(x, t)$ is the equilibrium distribution function. The functionality of the equilibrium distribution, $f_{i}^{e q}(\underline{x}, t)$, in the last term of Eq. (3.1) is chosen to ensure that both mass and momentum are conserved. Furthermore, $f_{i}^{e q}(\underline{x}, t)$ is quadratic in the fluid velocity and therefore accurately captures fluid inertial effects. In the limit of small Mach and Knudsen numbers, one can perform a Taylor series like expansion on Eq (3.1). and recover the Navier- 
Stokes equations of motion; therefore, this approach is directly applicable to microfluidic systems.

The single particle distribution function is a function of "lattice" velocity, $i$, position, $x$, and time, t. Eq. (3.1) is evolved on the lattice. $f$ is a moment baring function and contains all of the important fluid information locally at each lattice site. The zero ${ }^{\text {th }}$ discrete moment,

$$
\rho=\sum_{i} f_{i}(\underline{x}, t)
$$

gives the local fluid density, where $\rho$ is the fluid density. The first discrete moment with respect to the lattice velocity, $e_{i}$, gives the local momentum density,

$$
\rho \underline{u}=\sum_{i} f_{i}(\underline{x}, t) e_{i}
$$

Finally, the velocity distribution function can be used to calculate the force and torque acting on a stationary or mobile objects (of desired geometry) using Ladd's [2, 3] half-link model.

To enforce the no-slip condition at solid surfaces, the LB approach uses the simple bounce-back scheme. Specifically, if the $i^{\text {th }}$ component of $f$ is advected from a given fluid lattice site toward a solid phase lattice site then that component is reflected back to where it originated and collides with other incoming components of the distribution function at the originating lattice site. In this way, the presence of the solid phase is propagated back to the bulk fluid.

The bounce-back condition affords an extreme amount of flexibility. Specifically, the solid phase is accounted for in a logical array that includes all lattice sites in the system. If a lattice site is "true", i.e., solid phase, the bounce back condition is enforced. Otherwise, it is not enforced. As a result, it is very easy to include walls, obstacles and mobile particles into the capability. The user is only constrained by their ability to mathematically describe the object, i.e., to flag the appropriate lattice sites.

The lattice Boltzmann capability was constructed in a modular fashion. This permitted the inclusion of proposed physics modules. The modules included in this work, however, were chosen because they represented main categories of physical phenomena that are of primary importance to biological microfluidics, i.e., external field effects, intermolecular forces, electrokinetic phenomena, and non-Newtonian fluid models. Shown in the Figure 2 below is a diagram of the LB capability architecture. 


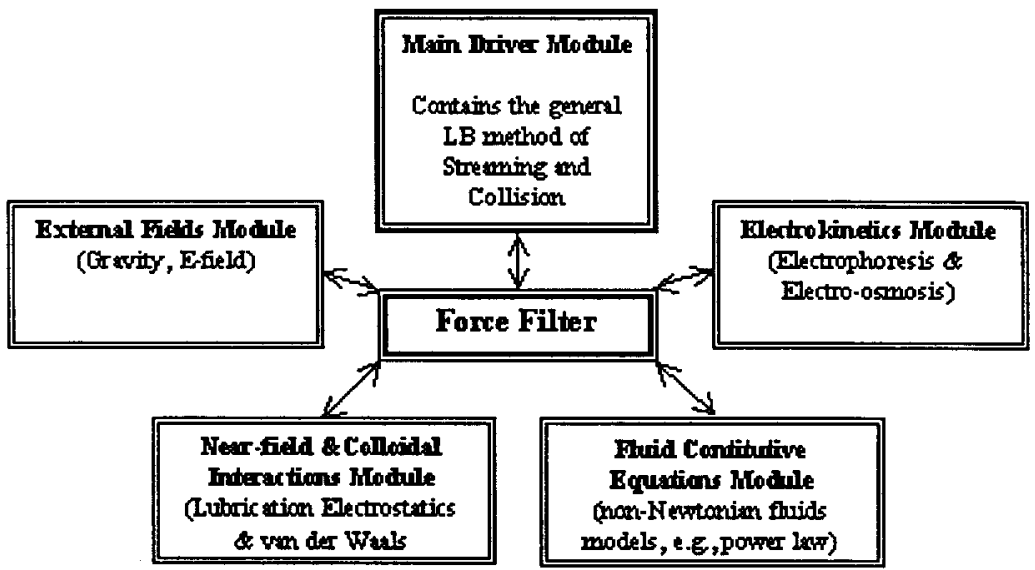

Figure 2 A basic block diagram showing the architecture of the module LB capability. The force filter, where time and length scales are converted from real space to lattice space and from lattice space to real space, is central to the overall capability.

While the LB method accurately handles all of the fluid mechanics, it calculates everything in "lattice space." As a result, hydrodynamic forces in lattice space need to be made dimensionless with the appropriate lattice parameters and converted to real space force units. This type of force conversion is commonplace in the lattice Boltzmann community. In the proposed work here, we will develop a set of conversion factors to convert real space forces to lattice space forces:

$$
\underline{F}_{L B}=\Psi_{\text {conv }} \underline{F}_{r}
$$

Here $\underline{F}_{\mathrm{LB}}$ is the force in lattice space, $\Psi_{\text {conv }}$ is the force conversion kernel and $\underline{F}_{\mathrm{r}}$ is the force from the module of interest in real space. This will permit the summing of all force contributions experienced by each particle in the flow configuration. During the initial stages of this work, we verified this simple relationship on a well known, controllable problem, namely, for a sphere sedimenting in a quiescent fluid (buoyancy force).

\section{External fields/Body forces/Dielectrophoresis}

In the presence of non-uniform electric fields, macromolecules are either attracted to local maxima or local minima in the E-field strength. This can be useful for separating biological macromolecules by collecting them, i.e., positive DEP, or separating them by physical properties through levitating macromolecules, i.e., negative DEP, into a fluid flow field. For parallel electrodes orthogonal, see Figure 3 below, to the flow direction placed at the base of a rectangular channel, the macromolecules for positive DEP are be attracted to electrode strip edges where the E-field strength is a maximum. 

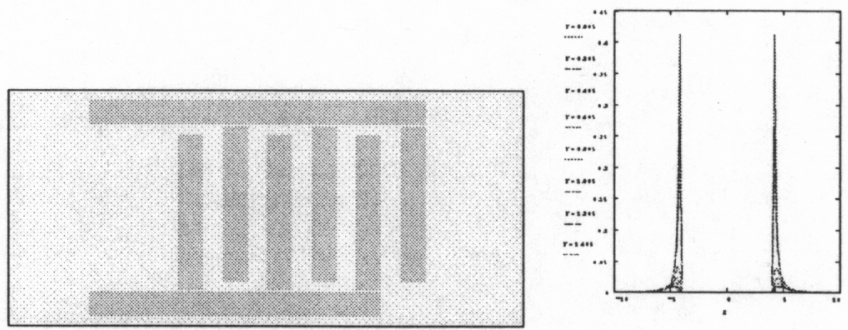

Figure 3 Left panel: Interdigitated gold electrodes. The flow is from left to right. The e-field strength is at a maximum at the electrode edges. The right panel shows the e-field strength at the edge of the electrodes as a function of applied voltage.

For negative DEP, particles are repelled (levitated) from regions of high field strength up into the flow field. Depending on the particle properties, differing species will be levitated to different positions in the flow field and therefore will separate from each other. This is known as field flow fractionation, or, FFF. The simplest form of the DEP force is given by the following expression:

$$
\left\langle\underline{F}_{D E P}\right\rangle=2 \pi \varepsilon_{1} a^{3} \operatorname{Re}\left[\left(\frac{\varepsilon_{2}^{*}-\varepsilon_{1}^{*}}{\varepsilon_{2}^{*}+2 \varepsilon_{1}^{*}}\right)\right] \underline{\nabla}(\underline{E} \cdot \underline{E}) .
$$

Where $a$ is the particle radius, $\varepsilon_{i}^{*}$ is the complex permittivity of the phase and $\underline{E} \cdot \underline{E}$ is the applied electric field intensity. The term in parenthesis is known as the Clausius-Mossotti factor. It is this factor, based on fluid properties and the frequency of the electric field that determines whether one has positive or negative DEP. This force is calculated in real space and passed through the force filter and converted to lattice space to be summed with the forces calculated using the LB method. While the DEP force is small in the "macroscopic" flow world, it is very important and useful in the microscope world of Bio-flips. Furthermore, The DEP flow cell to be studied in this proposal represents a fully integrated microfluidic subsystem that is relevant to several researchers and is proposed here to demonstrate the LB capability on this fully integrated microsystem.

To model such micro-unit operation we define a global coordinate and local coordinate systems, see Figure 4 below. 


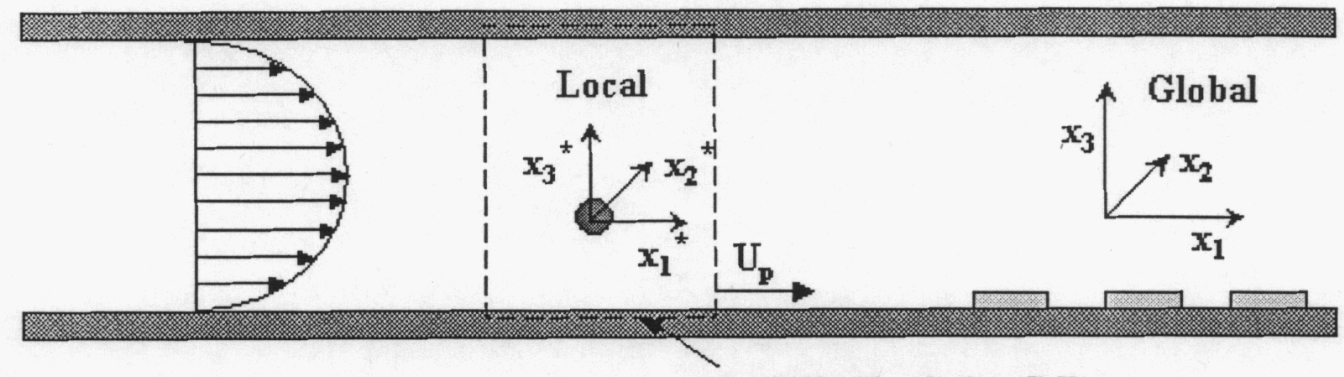

Periodic Simulation Cell

Figure 4 Typical Dielectrophoretic separator. The dashed rectangular region represents the simulation cell. The simulation cell moves with the target species in the flow direction toward the interdigitated electrode array, denoted by gold rectangles.

Here the DEP separator is described in full in the global coordinate system and the target species is tracked in the local simulation cell coordinate system, the simulation cell is denoted in dashed lines with the particle at the centriod. The simulation cell translates with the particle as it approaches the electrode array. The simulation cell has periodic boundary conditions; therefore, this "single" particle simulation set-up actually takes into account dilute concentration effects through the target species periodic images.

\section{Intermolecular/Colloidal force interactions}

Intermolecular/Colloidal force interactions act in a pair wise fashion (two body near-field interactions); therefore, when particles are within sufficient range of each other $(<1 \mathrm{~mm}$ for electrostatics and $<100 \mathrm{~nm}$ for van der Waals) we sum their force contributions with the forces calculated by the LB. Specifically, we use DLVO theory to calculate these forces pass them through the force filter, see Eq. (3.4), and sum the result with the calculated LB force.

The approach to incorporate these forces is precisely the same approach that the PI uses to correct for near-field hydrodynamic interactions for lubrication forces. Following the same approach, the non-hydrodynamic forces are summed with the PI's hydrodynamic force correction to get the total force correction for a particular particle:

$$
\left[\begin{array}{l}
\delta \underline{F} \\
\delta \underline{L}
\end{array}\right]^{T}=\left[\begin{array}{l}
\delta \underline{F} \\
\delta \underline{L}
\end{array}\right]^{H}+\left[\begin{array}{l}
\delta \underline{F} \\
\delta \underline{L}
\end{array}\right]^{N H},
$$

where the superscripts $T, H$, and $N H$ represent the total force, the near-field hydrodynamic force, and non-hydrodynamic force corrections respectively. This generalized framework permits the rapid inclusion of additional intermolecular/colloidal forces. 
All biological macromolecules experience intermolecular force interactions with each other in solution, e.g., repulsive electrostatic and attractive van der Waals force interactions. These force interactions can cause non-intuitive particle behavior particularly when there are many interacting particles in confined domains, e.g., particle aggregation, wall adhesion, etc. In microscale channels these force interactions become increasingly more important. Electrostatic interactions act on the length scale of the Debye screening length, which is a measure of the thickness of the electric double layer, i.e., electrostatic force interactions act over a distance usually less than one micron. Where the Debye screening length, $\kappa^{-1}$, is inversely proportional to the square root of the ionic strength of the solution:

$$
\kappa^{-1}=C / \sqrt{[\text { Electrolyte Concentration }]},
$$

where $C$ is a constant dependent on the salt species in solution [4]. The units on Eq. (5.2) are nanometers, $\mathrm{nm}$. The attractive van der Waals forces on the other hand act on the length scale of $100 \mathrm{~nm}$ or less [4]. Because both of these force interactions act in a pair wise fashion (two body near-field interactions), their presence is accounted for in form of an interaction potential, which provides a framework for inclusion of other pair wise force interactions that are of interest. These force interactions are present, and in micro-flows, they must be taken into account.

\section{Phoretic transport mechanisms}

Electrophoresis and electro-osmosis transport mechanisms are very common in all microfluidic systems. The mechanism that causes each phenomenon is the same in both cases. Specifically, a charged surface in an electrolyte solution has a cloud of counter ions that seeks to electrically neutralize the surface, or, achieve electro-neutrality. The charged surface and the cloud of counter ions are what is known as the electric double layer. When subject to a uniform field, usually a DC field, the electrode with the same charge as the counter ion cloud causes a flow by repelling the like ions. For stationary surfaces this gives rise to electro-osmotic flows, and for mobile surfaces, this gives rise to electrophoretic motion of the mobile species. The velocity of counter ion repulsion can be described as a slip velocity at the charged surface [5]. For electrophoresis and electro-osmosis the slip velocity is given by

$$
u_{s}=\frac{\varepsilon \zeta}{4 \pi \mu} E_{s}
$$

Where $u_{\mathrm{s}}$ is the slip velocity at the surface, $\varepsilon$ is the permittivity of the suspending fluid, $\zeta$ is the zeta potential (the measured surface potential with adsorbed counter ions), and $E_{\mathrm{s}}$ is the applied electric field. This velocity is the electrophoretic velocity at which a particle with zeta potential $\zeta$ travels through a fluid of permittivity of $\varepsilon$ and viscosity of $\mu$ subject to a uniform electric field evaluated at the surface, $E_{\mathrm{s}}$. For a stationary surface, Eq. (6.1) describes the boundary condition at a charged surface that drives electro-osmotic flow. Other phoretic transport phenomena, such as, diffusiophoresis and thermophoresis, can also be described in terms of a slip velocity [5]; therefore, this represents a general framework for including this class of transport phenomena. 
While the physics above appears somewhat disjoint, their sum represents the coupled phenomena that exist in many real Bio-fluidic-chips. For example, it is not uncommon to use dielectrophoresis to trap or sort biological macromolecules. In these micro-systems, biomolecules convect with the fluid in microchannels. The bio-molecules in particle-laden flow have three predominant effects: External fields, i.e., gravity and $\mathrm{AC}$ electric fields, are incorporated into the LB method through analytic and semi-analytic expressions [6]. The forces induced by these two external field effects, buoyancy and DEP forces, will also be passed through the force filter and summed with the force as predicted by the LB method, see Eq. (3.4) above. Additionally, the resulting capability is 3D and parallel, using F90 and MPI.

\section{Results and Discussion}

The resulting effort was directed by the needs of the BioFlips PIs; therefore we focussed in the three following areas:

1. Dielectrophoretic separations achieved by preferential particle trapping,

2. Particle transport independent of fluid motion, traveling wave Dielectrophoresis, and

3. Suspension properties in microflows, sudden contraction in microflows.

In addition to these three thrust areas, we explored some anamolous effects experience in DEP separations. In particular, we explored and elucidated the effects of the electric double layer on the polarizability of the target species.

\section{Dielectrophoresis}

As discussed above, Dielectrophoresis, DEP, arises as a result of an induced dipole in a nonuniform electric field, which results in a net Lorenz force either levitating or attracting the target species [7]. Several PI's in the BioFlips program employ DEP to perform micro-separations and sample preparation. Shown in Figure 5 below is the time average DEP force for standing wave DEP. 

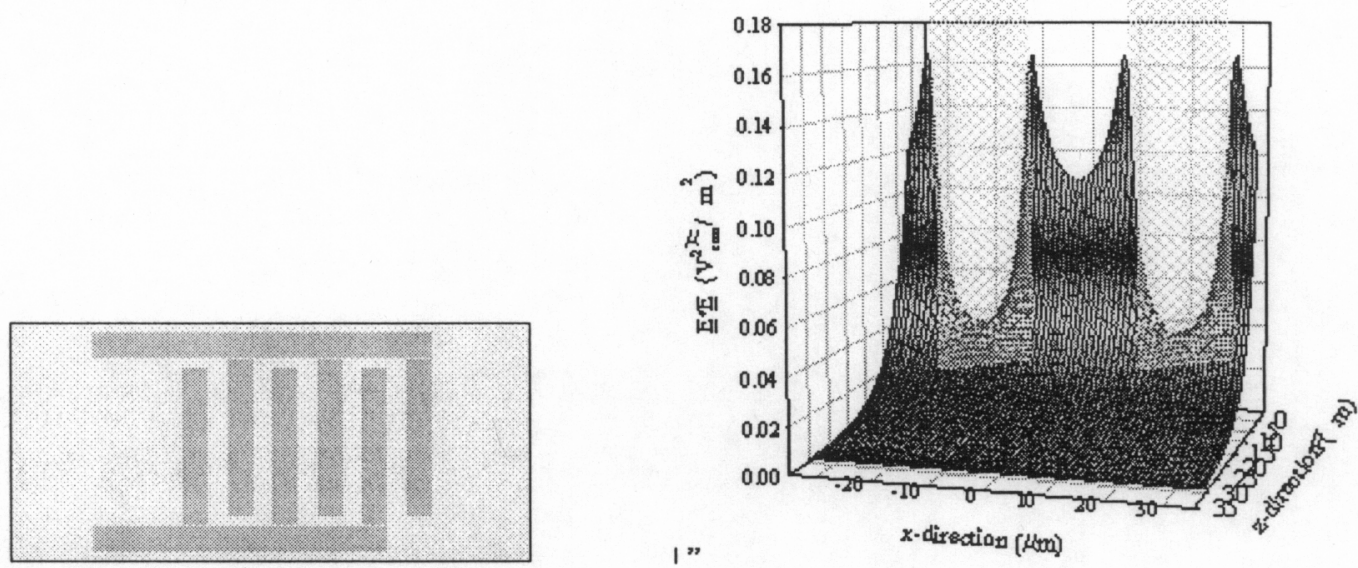

Figure 5 Interdigitated electrode configuration and the electric field intensity produced by this electrode configuration. The gold rectangles in the right panel represent electrodes in the array. The surface plot of electric field intensity emanating from the electrode surface.

The DEP force is directly proportional to the gradient in the electric field intensity, $\underline{E} \cdot \underline{E},[8]$ as shown in the right panel of Figure 5. Given that the electric field intensity is a maximum at electrode edges, the DEP force is also a maximum at electrode edges, see Figure 6.
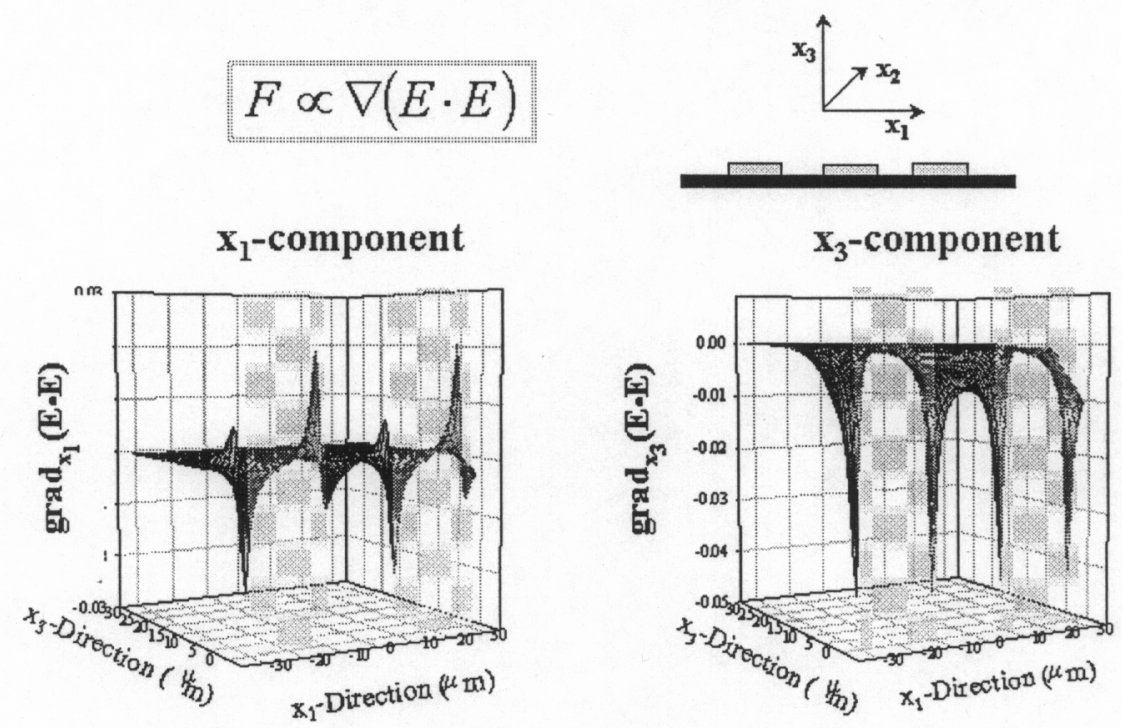

Figure 6 Gradients in the electric field intensity. The left panel is the gradient in the flow direction and the right panel is the gradient in the vertical direction.

The gold rectangles represent the electrodes and the $z=0$ surface or floor of the electrode array. Note that the gradients are indeed much stronger at the electrode edges. The $\mathrm{z}$-direction or $\mathrm{x}_{3}$ direction is always negative, the vector pointing to the electrode edge. When the Clausius- 
Mossotti factor is "positive" we have postive DEP and the target species is attracted to regions of high field intensity, toward the elctrode edge. When the Clausius-Mossotti factor is "negative" we have negative DEP and the target species is repelled or levitated due to the presence of the applied field.

To assist designers, we explored the effects of changing the electrode width and spacing on the magnitude of the gradient in the electric field intensity, [8], see Figure 7

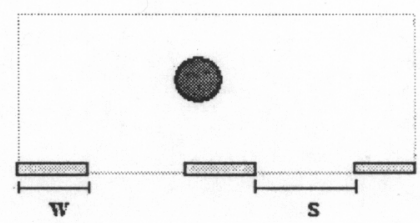

Dependence on applied voltage

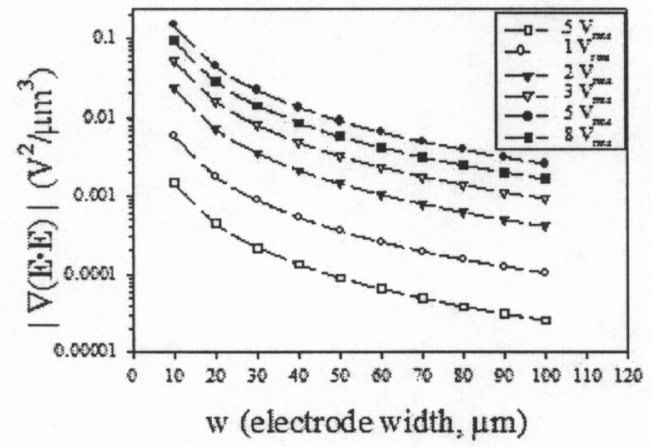

Dependence on spacing between electrodes

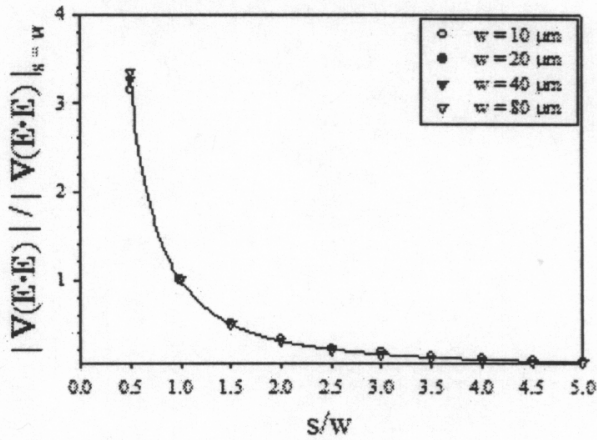

Figure 7 The dependence of the magnitude of the gradient in the electric field strength. The left panel is the dependence on the applied voltage. The right panel is the dependence on the spacing between electrodes.

As shown the gradient in the electric field intensity steepens as the electrode width is reduced. Also shown on the right hand panel, the gradient in the electric field intensity increases as the electrodes are brought closer together. For further details the reader is referred to [8].

To determine the critical Reynolds number of operation for a given applied voltage, we performed a serious of numerical experiments to construct a phase diagram, see Figure 8 below. 

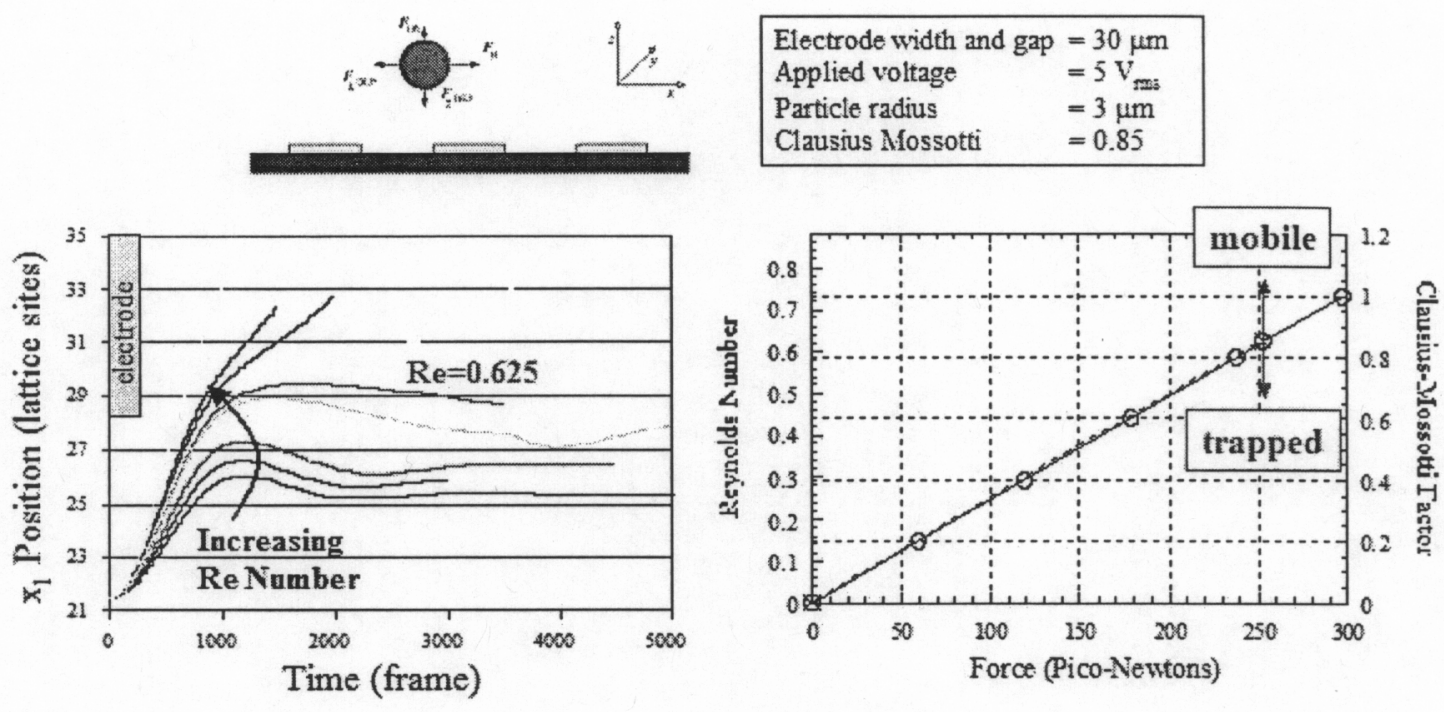

Figure 8 Determination of the critical Renolds number for a given electrode configuration, applied voltage and particle radius. The left panel shows particle translation as a function of Reynolds number relative to an electrode, denoted in gold. The right panel is a phase diagram for predicting particle trapping given a Clausius-Mossotti factor and a channel Reynolds number.

To assist designers to know what flow rates or Channel Reynolds numbers that they can operate their separators, we performed a series of simulations to determine the critical, channel Reynolds number as a function of Clausius-Mossotti factor for target species trapping. By doing a series of experiments, we were able to construct a phase diagram, see right panel, for rapid estimation of DEP separator operating conditions, $[9,10]$.
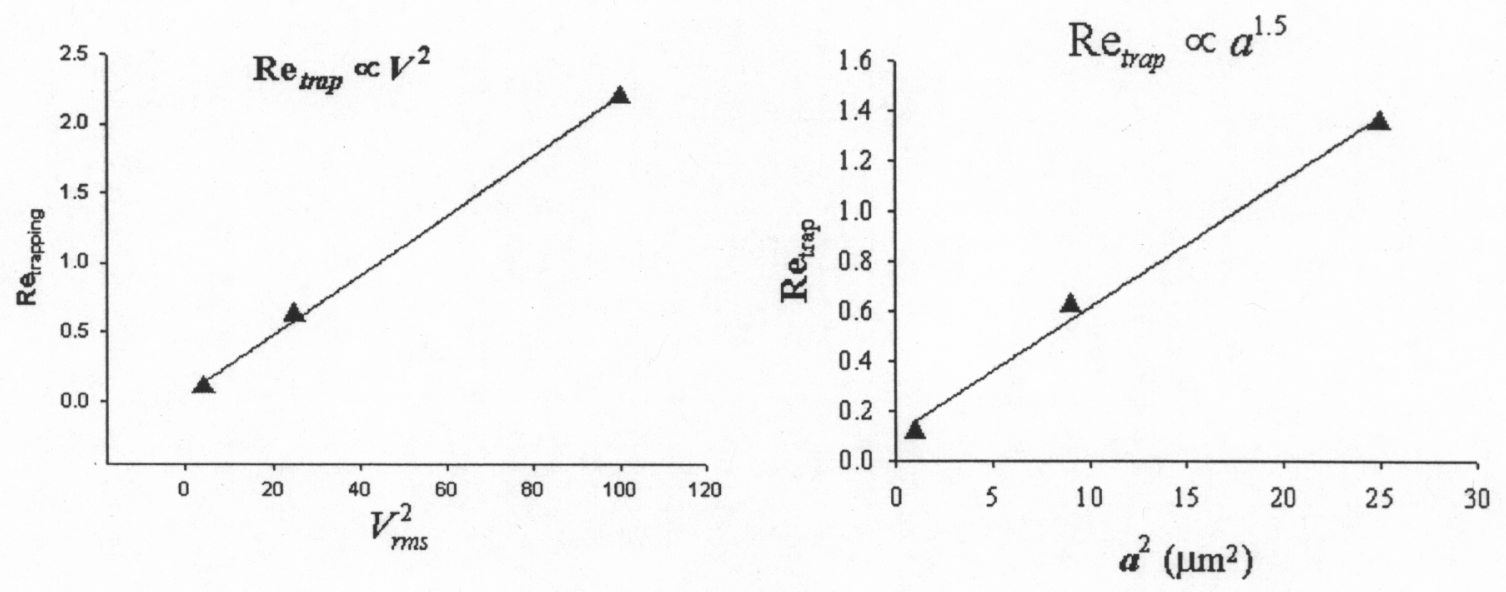

Figure 9 Critical Reynolds number for particle trapping as a function of applied voltage (left panel) and as a function of target species radius, a (right panel). 
In addition to these studies, we also validated our dynamic simulation capability with theory by predicting the dependence of the critical Reynolds number on the square of the applied voltage and the target species radius. Our simulations exhibited the expected dependence. Two additional parameters that practitioners need to know are 1) how long does the electrode array need to be to and 2) what depth of channel should be used for an applied voltage to ensure target species manipulation/trapping; therefore, we performed a series of numerical experiments to study the depth of penetration of the Dielectrophoretic force in a DEP separator, see Figure 10.

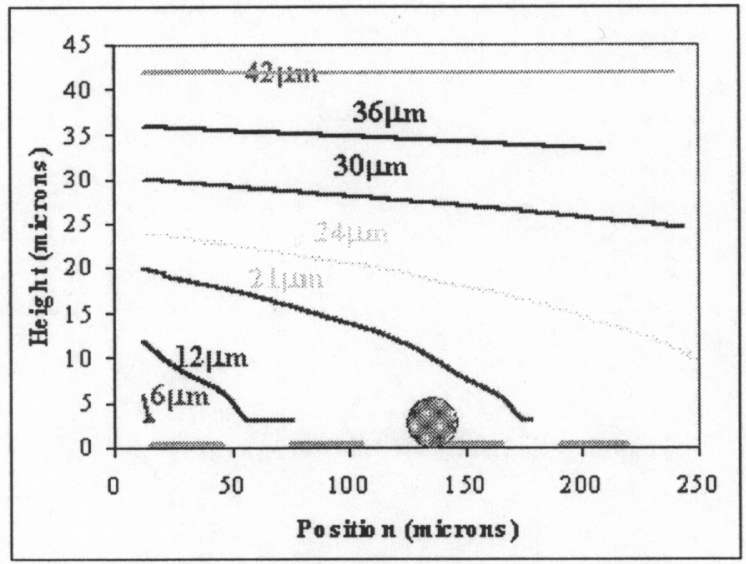

$$
\begin{array}{ll}
\text { Neutrally buoyant particle } & \\
\text { Reynolds number } & =0.5 \\
\text { Particle radius } & =3 \mu \mathrm{m} \\
\text { Applied voltage } & =5 \mathrm{~V}_{\text {rms }} \\
\text { Clausius Mossotti factor } & =0.85
\end{array}
$$

Figure 10 Dielectrophoretic particle trapping as a function of particle height for a given applied voltage, electrode configuration, Clausius-Mossoti factor and species radius.

For the conditions show on the right side of Figure 7 the DEP field reaches approximately 40 um into the fluid. Therefore in the design of a separator that operates at similar operating conditions, the user will need to adjust the depth of the separator channel and the length of the overall electrode array to ensure that the species of interest is trapped with high efficiency out of the sample.

To make this unique capability more useful, we extended it to include multiple species. This was as a result of our collaboration with MD Anderson cancer center. In our support of their effort, we developed capabilities to predict species cross over frequencies and to predict suspension effects on separator performance. In the MD Anderson application they were particularly interested in separating the various white cells from whole blood samples to monitor changes in concentration in response to exposure to pathogen. The cross over frequencies, where the Clauisus-Mossotti factor is zero, for T and B Lymphocytes, Monocytes and Granulocytes are shown in Figure 11. 


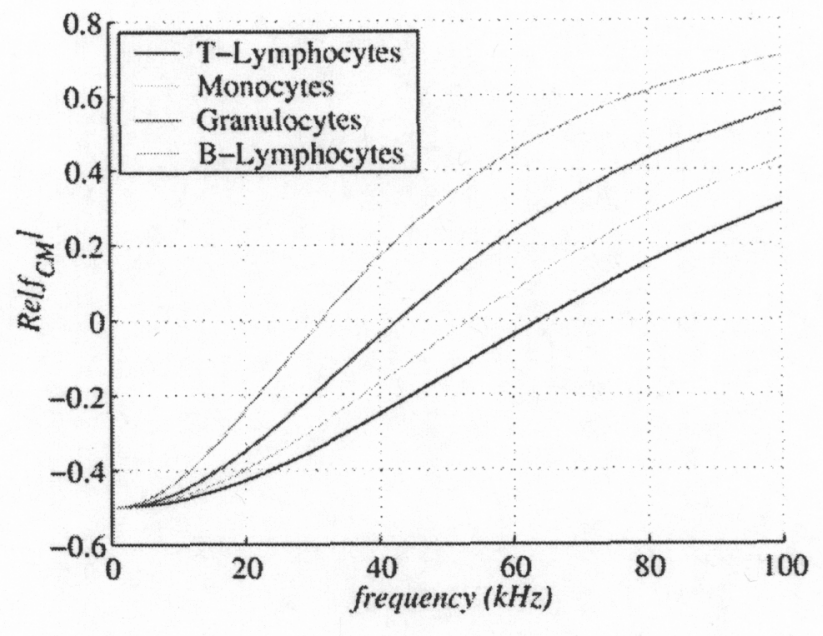

Figure 11 Dielectrophoretic response of white blood cells. The goal is to determine the cross over frequencies to optimize the Dielectrophoretic separation protocol.

By knowing the cross over frequencies, the DEP separator protocol can be optimized to effect desired separations. Finally, we developed a multi-species DEP simulation capability, see Figure 12.
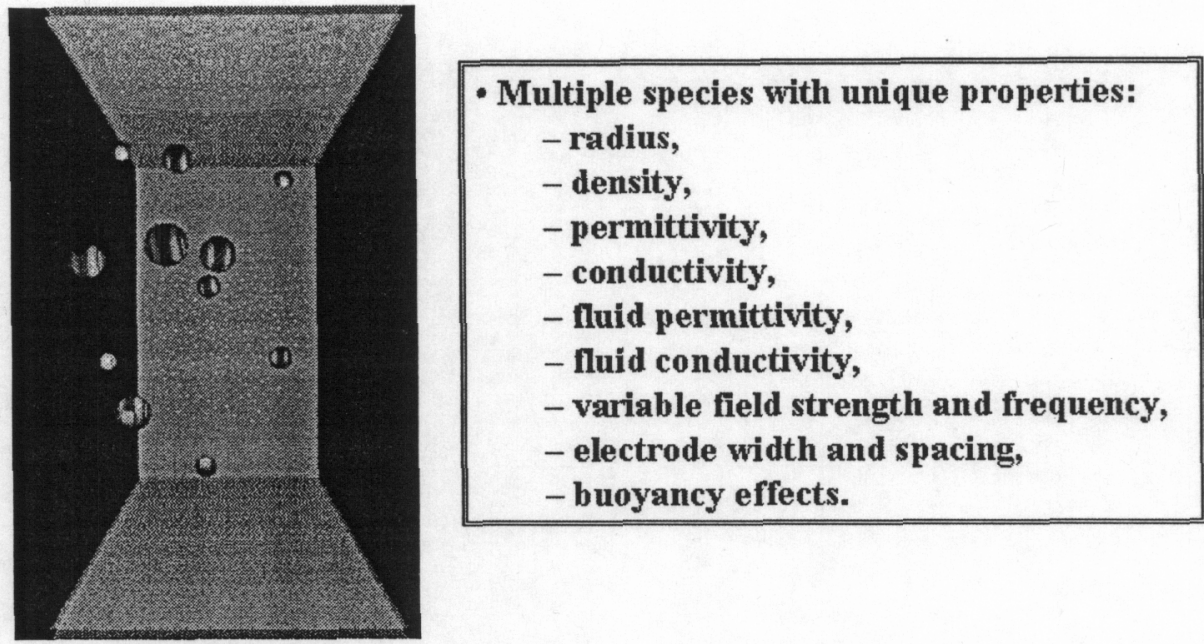

Figure 12 Multispecies simulation capability. Species in solution can be attributed with unique physical properties, e.g., Dielectrophoretic properties. 
In this unique capability, which uses the simulation configuration as described in Figure 4, researchers can now explore separation protocols as a function of the species present, fluid conditions and applied voltages.

With this capability, we explored the design of a traveling wave DEP separator with Professor Rosemary Smith at UC Davis. Shown below in Figure 13 is a cartoon of their separation chamber.

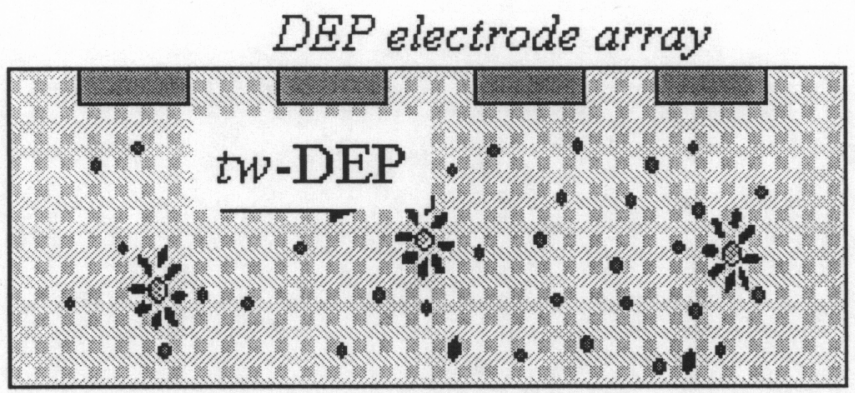

Figure 13 Traveling wave Dielectrophoretic separator. Antibody analyte aggregates translating independent of the fluid to a detection region.

In the above figure, a sample of body fluid is drawn into a chamber of quiescent fluid and liposomes form aggregates mediated by target analyte in solution. The liposome aggregates are translated independent of fluid flow by traveling wave DEP to a detection region. The traveling wave force is dependent on both the standing wave and the gradient in the phase of the applied field as shown in Figure 14.

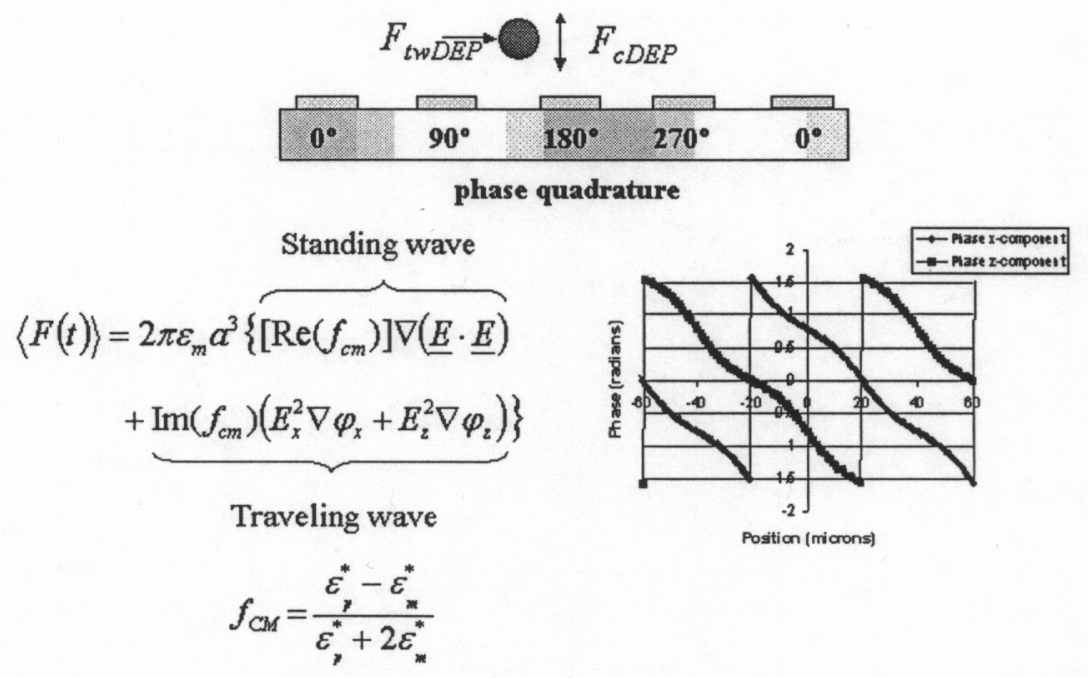

Figure 14 Traveling wave Dielectrophoresis translate the target species independent of the fluid (top). The driving force is the gradient in the phase of the electric field (right panel). 
Note that the time averaged force contains both the standing and traveling wave components; therefore, the target species is levitated and translated during traveling wave DEP. Also note that the traveling wave piece is dependent on the imaginary part of the Clausius-Mossotti factor, $f_{C M}$. To calculate the translocation velocity, we determined the particle velocity that is equivalent with the tw-DEP force. In Figure 15 below, a plot of the translocation velocity as a function of height above the electrode array is given.
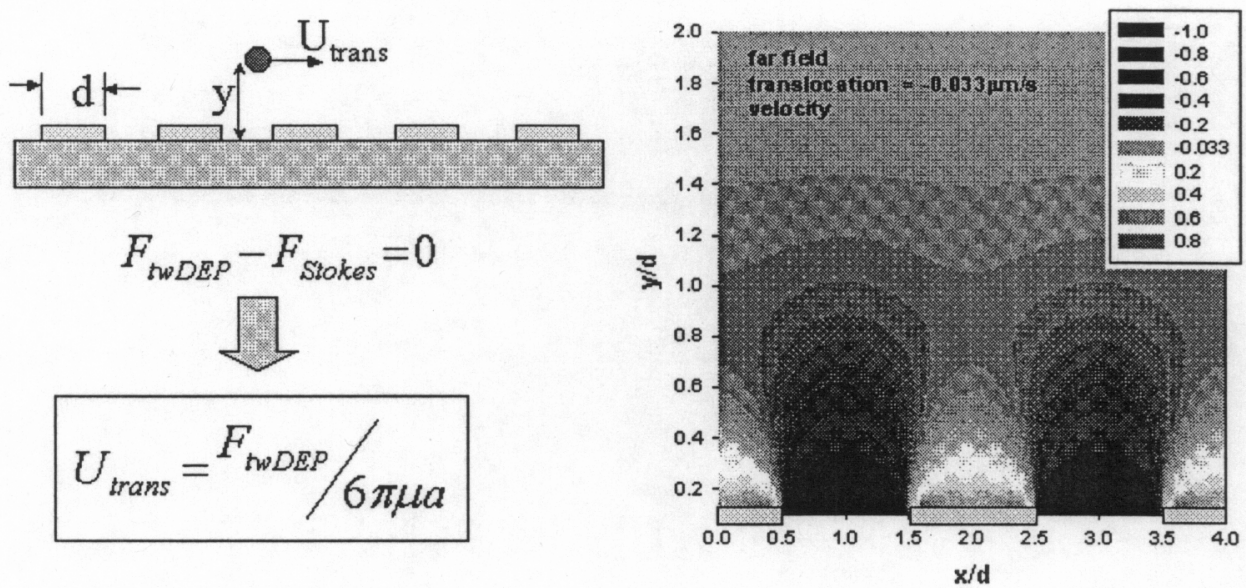

Figure 15 Translocation velocity of target species predicted from the balance of the traveling wave force and the viscous force exerted by the fluid on the body. The right panel is a map of the resulting traveling wave velocities as a function of position from the electrode array.

As shown by Green et al.,[11] there exists an optimal height above the electrode for traveling wave DEP. This critical height is when the ratio of the height with the electrode width is $\sim 1$. In direct support of the UC Davis effort, we determined translocation velocities for various particle sizes and applied voltages, see Figure 16 below.

\section{Translocation Velocity $(\mu \mathrm{m} / \mathrm{min})$}

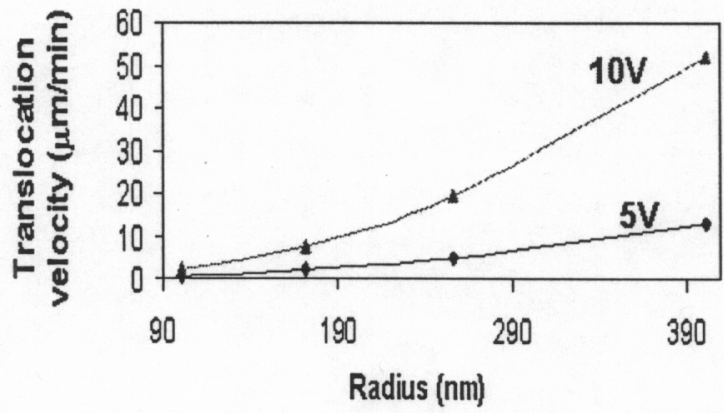

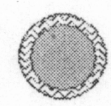

$\mathrm{d}=10 \mu \mathrm{m}$

$\mathrm{h}=2 \mathrm{~d}$ $150 \mathrm{mM}$ soln

$\varepsilon_{\mathrm{m}}=80$

Figure 16 Predicted translocation velocities as a function of particle size and applied voltage. 
In this study we chose a physiological solution conductivity and calculated translocation velocities at various aggregate radii for two applied voltages. These studies directly impacted the UC Davis device design.

\section{Double layer effects}

A common phenomenon kept reappearing in our support of both MD Anderson and UC Davis (as well as other efforts that use DEP). This phenomenon was that target species tended to exhibit the opposite DEP behavior. More specifically, the calculated Clausius-Mossotti factor would predict the sign of the DEP force acting on the target species, but the species would respond as if the Clausius-Mossotti factor had an opposite sign (while the magnitude of the force was not necessarily equal and opposite to what was predicted; therefore, we looked into this phenomenon. We noted that while DEP is a volumetric force that the Clausius-Mossotti factor depeneded heavily on the surface properties of the target species; furthermore, we observed that the species that exhibited this phenomenon had a surface charge and therefore an electric double layer. This led us to develop a new approach for calculating the Clausius-Mossotti factor to take into account not only the Stern layer but the diffuse double layer as shown in Figure 17.

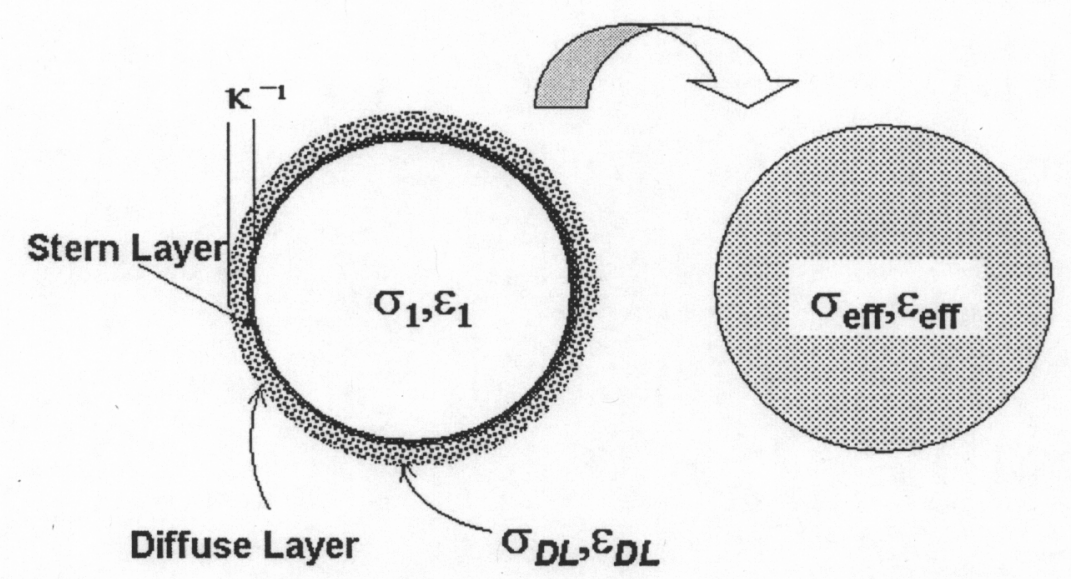

Figure 17 The details of the electric double layer incorporated into a respesentative particle with the appropriate effective conductivity, $\sigma$, and effective dielectric, $\varepsilon$, properties.

The electric double layer consists of two parts, 1) the bound counter ions on the surface, the Stern layer and 2) the diffuse counter ion cloud that seeks to neutralize the charge of the surface, a condition known as electro-neutrality. By calculating the conductivity and the change in permeability in the double layer and using the shell model [7], we incorporate the presence of the electric double layer when predicting the effective properties of the target species. As it turns out, all biological species, e.g., analytes, and polystyrene, which is ubiquitous in microfluidics all have a surface charge and therefore exhibit anomalous response to DEP forces. Therefore such an understanding and predictive capability is critical to the successful implementation of DEP to 
effect desired separations. Many of those who are new to the use of DEP erroneously just switch the sign on the Clauisius-Mossotti factor to account for the unexpected change in behavior. This has occurred even within the BioFlips efforts when predicting the DEP response of polystyrene beads. In Figure 18 below, typical properties for polystyrene beads used in biological applications are given.

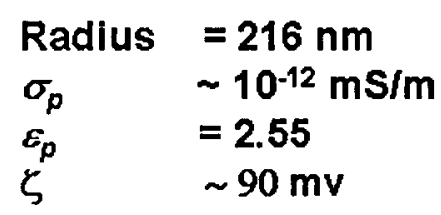

Hughes et al., 1999

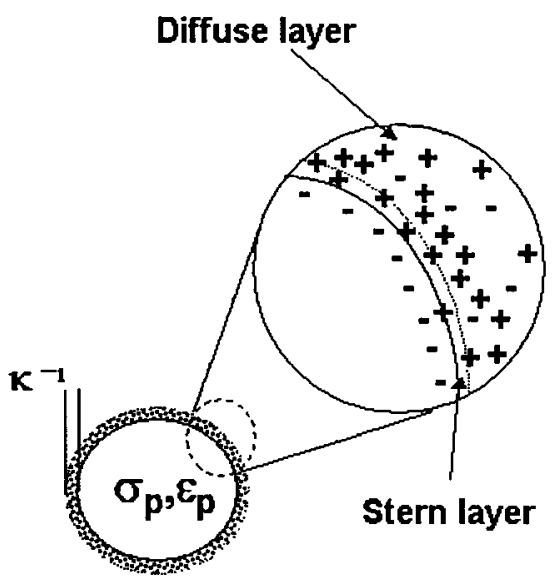

Figure 18 Polystyrene bead. Physical properties taken from Hughes et al., 1999.

As shown, we assumed a radius of $216 \mathrm{~nm}$. Also shown is a cartoon of the electric double layer. Given these properties, we applied our new capability to predict changes in the ClauisusMossotti factor as a function of ion strength of the solution. The results for three solution conductivities, concentrations of $[\mathrm{NaCl}]$ are given below in Figure 19. 


\section{Constant Zeta potential, variation in concentration}

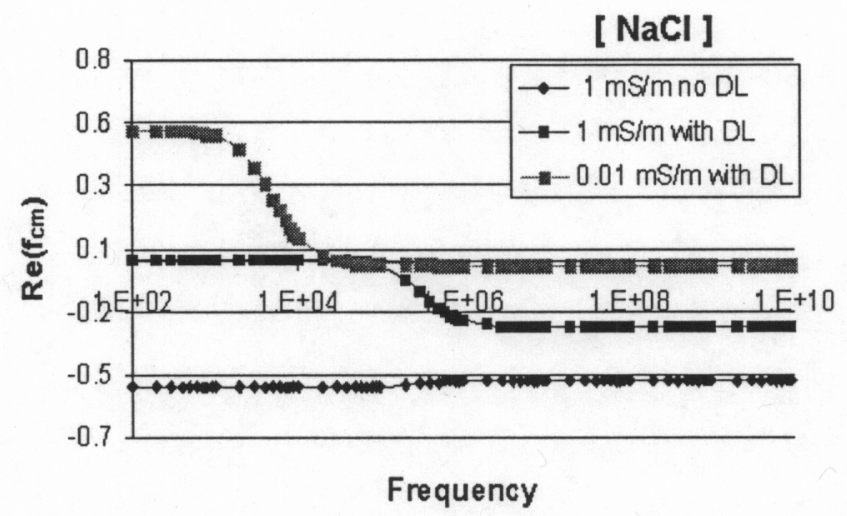

Figure 19 The real part of the frequency dependent ClausiusMossotti factor for Polystyrene as a function of ionic strength of the solution.

Without accounting for the electric double layer, one would always predict a negative ClausiusMossotti factor and therefore would always predict a repulsive DEP force. In practice however, polystyrene beads are "weakly" trapped depending on the ionic strength of the solution. As shown above in Figure 19, our new approach predicts the "positive" DEP as a result of double layer effects. Also note that the "cross-over" frequency decreases with increasing solution conductivity.

To explore the effects on simulants, such as, Avidin, we also made predictions todemonstrate the new capability on proteins, see Figure 20.

Protein

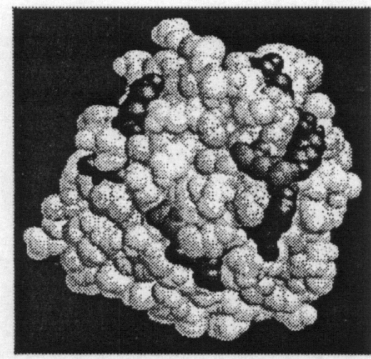

Figure 20 Typical properties of proteins.

\section{Size Range}

$\sim 10 \mathrm{~nm}$

\section{Zeta Potential}

- Dependent on pH

- $\zeta \in[60 \ldots-60 m v]$

[Wiącek and Chibowski, 2002] 
In is important to note that the Zeta potential, which is extremely important in estimating the conductivity and permittivity of the electric double layer, is highly sensitive to the solution $\mathrm{pH}$.

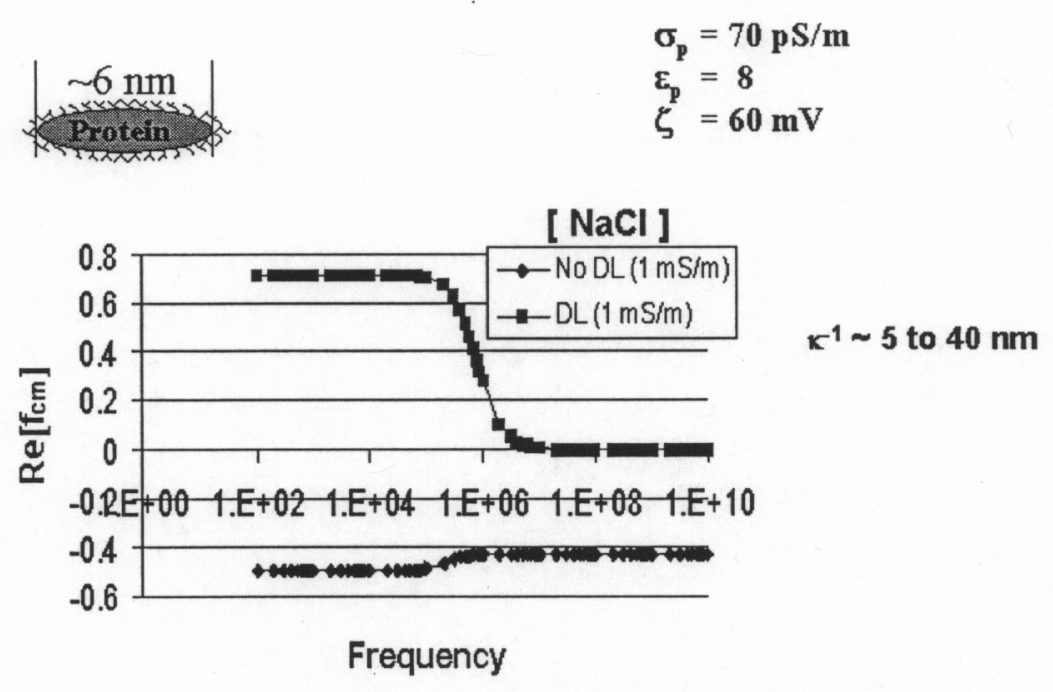

Figure 21 The real part of the Clausius-Mossotti factor for a typical protein at two different fluid conductivities.

Again, in Figure 21, we see the dramatic effects of the electric double layer on the DEP response of a protein. Consistent with the typical prediction of the Clausius-Mossotti factor, the response without taking into account the effects of the electric double layer always gives negative DEP; however, when accounting for the electric double layer, the response is significantly different, and in the case shown, the response now always predicts positive DEP. We preformed this analysis specifically for the UC Davis team because they were using Avidin as their simulant.

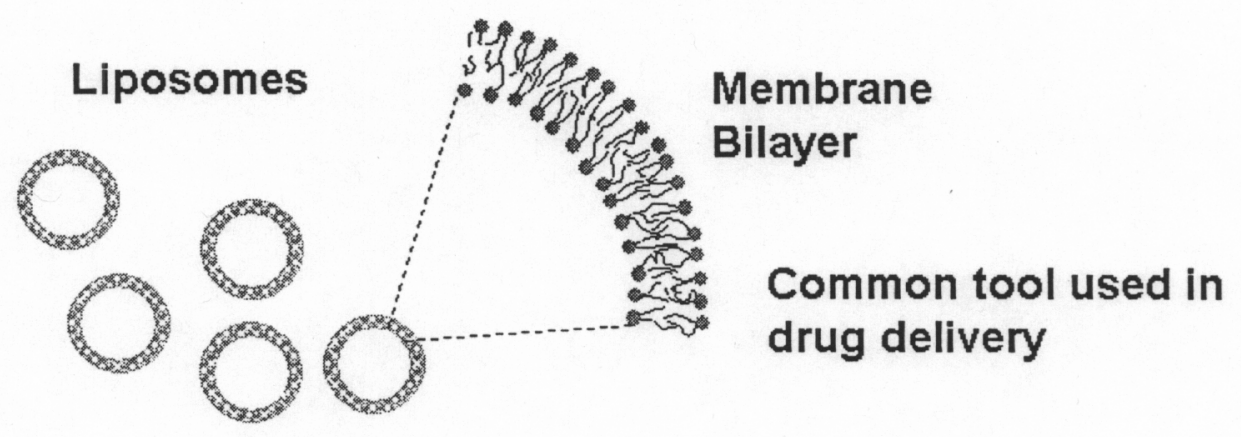

Figure 22 Liposomes with a manufactured biolayer.

A liposome is simply a spherical volume of fluid enclosed by a bilayer. 


\section{Radius $=500 \mathrm{~nm}$ Membrane Properties Capacity $=6.7 \mathrm{mF} / \mathrm{m}^{2}$}

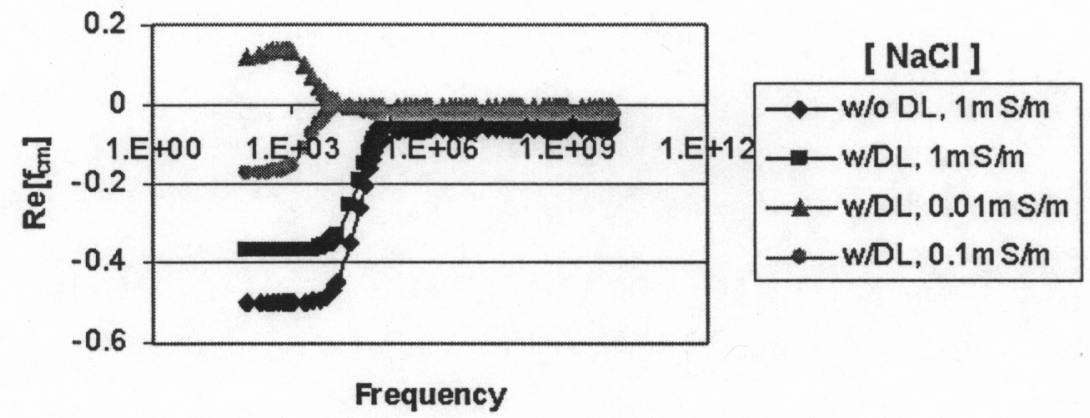

Figure 23 The real part of the Clausius-Mossotti factor for a typical protein at two different fluid conductivities.

The Liposomes have very unique and interesting behavior. Given that the fluid inside the liposome is identical to the exterior fluid, one would expect relatively uninteresting behavior. As shown, however, the behavior is quite interesting. Note how the response switches from negative to positive DEP in the low frequency range when the fluid conductivity is less than $0.1 \mathrm{mS} / \mathrm{m}$. This elucidates the fact that while DEP is a body force the species response is predominantly governed by the electrical properties of the membrane.

With this new capability, device designers and practitioners can more accurately predict the response of "charged" species and be able to tune fluid properties to achieve desired separations.

\section{Suspension Mechanics}

One of the beauties of the lattice Boltzmann method is the ability to handle multiple suspended species with various properties. In addition, one of the main foci of the BioFlips program had to do with sample collection and fluid delivery. In particular, at the South Carolina meeting, there was a focus group on micro-needles. Also, we had interacted with UCB on drug delivery and microneedles. It became apparent that there were issues that needed to be understood and overcome to unleash the use of microneedles in real world applications. In particular, in the UCB case, they were experiencing microneedle clogging at very low volume fractions $(0.1 \%)$, see Figure 24 below. 

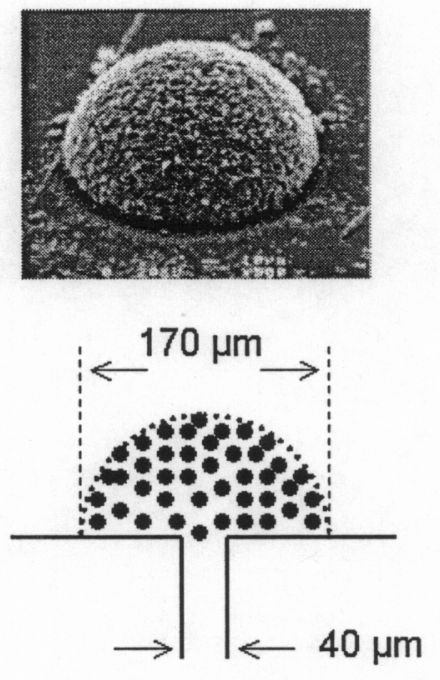

- $\sim 1 \mu \mathrm{m}$

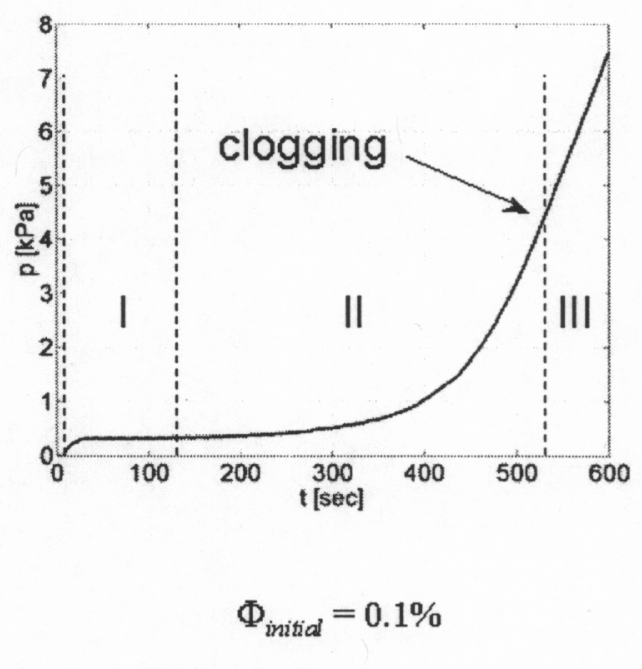

Figure 24 Experimental results of particle coagulation through a sudden contraction (Results curtesy of Professor Dorian Liepmann).

On the left the length scales of interest are shown, and on the right is an experimental plot showing the applied pressure as a function of time to elucidate the onset of clogging or particle coagulation. To assist the UCB team, we developed simulation capabilities to study the fluid mechanics and suspension behavior through a sudden contraction. As shown in Figure 25 below, we developed a new capability to apply a pressure gradient through the contraction to study the fluid velocity fields, shear-stress fields and pressure field[12]. 

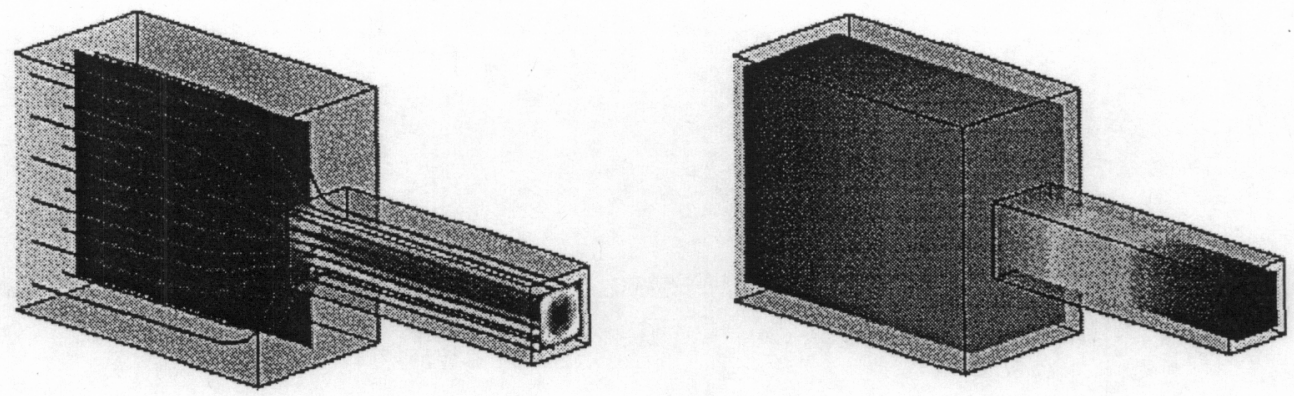

Figure 25 Lattice Boltzmann representation of sudden contraction (3:1 z-direction, 2:1 y-direction) in a microneedle. The left panel represents the velocity field and the right panel is the magnitude of the pressure field.

Due to conservation of mass and volumetric flow rate, we see a significant increase in fluid velocity through the contraction. This is not necessarily earth shattering, but what is important to note is that the stream-lines leading to the contraction exhibit a significant fluid acceleration that impart sufficient momentum to the suspended species to cross stream lines. The 1 micron beads used had a polymer surface coating to disrupt the electric double layer, but this surface coating serves as a hydrophobic corona that mediates particle coagulation when particles come close to contact. As a result, due to the contraction, the particles cross stream-lines and interact and coagulate. Also shown is the pressure field, it is interesting to note that the pressure field has a much more significant gradient in the narrow portion of the micro-needle. While this is intuitively obvious, this result graphically demonstrates this physical phenomenon. With the LB method, we can easily vary the channel geometry; therefore, this capability is ideal for exploring and optimization of micro-needle design.

In keeping with the specific aims of the proposed effort, we developed the capability to handle suspensions in microchannels, from dilute to concentrated suspensions, see Figure 26 below for a typical simulation cell at $40 \%$ concentration by volume.
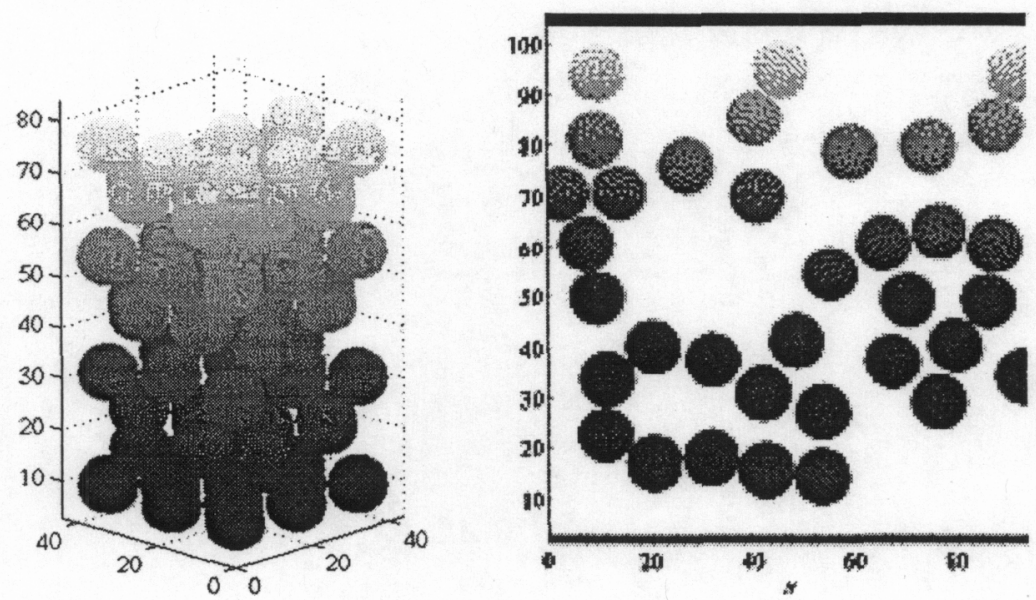

Figure 26 Lattice Boltzmann capability to handle concentrated suspensions. The left panel is the suspension initialization at a specific concentration. The right panel is a snap-shot of particle positions in a rectangular channel in pressure driven flow. 
Also, shown is a frame from a dynamic simulation of a suspension in a straight channel. Here each particle position is tracked and the particles are fully coupled with each other and the environment, that is to say they are interacting hydrodynamically with each other and the channel walls with no approximation. The color in both panels is insignificant; it is just an artifact of MatLab. Our capability not only accounts for far field hydrodynamic interactions, but we have lubrication interactions built in for particle-particle and particle-wall interactions.

To validate our new capability, we compared our suspension results with the gold standard put forward by Nott and Brady [13]. They discovered unique ways to characterize suspension flow between parallel walls. In keeping with their developments in Figure 27 below we characterize our results for suspension behavior.
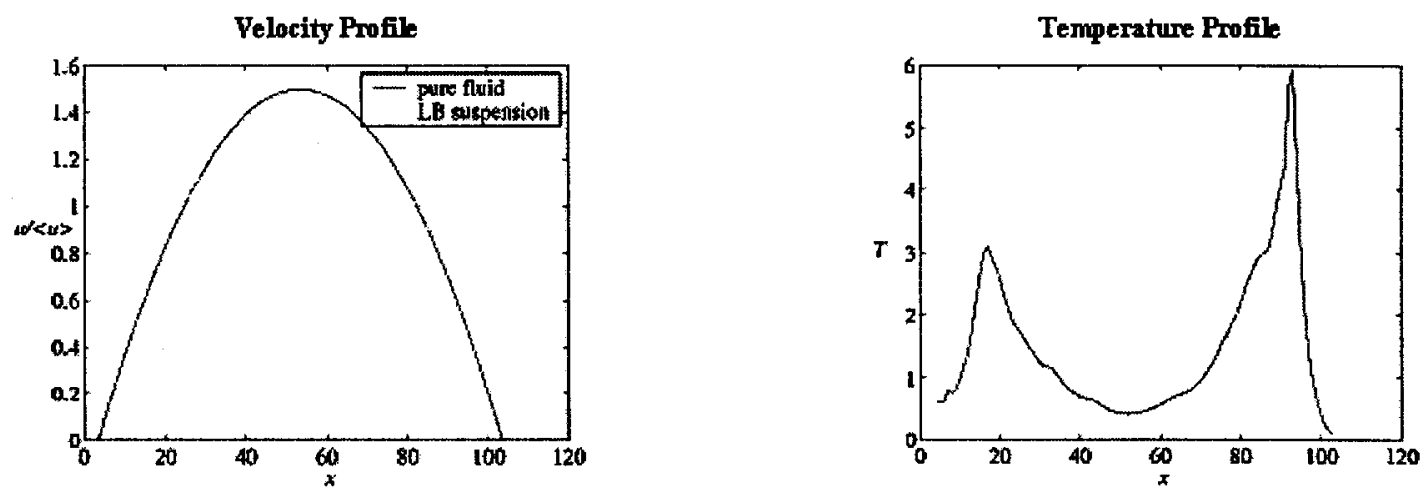

Figure 27 Suspension velocity profile compared with a pure fluid velocity profile (left panel), and the collision frequency, e.g., suspension temperature, on the right.

In the left panel we compare the suspension velocity with the velocity profile for pure fluid. The observed behavior is best understood by taking into account the finite size of the suspended species. Note the violation of the no slip condition on the walls, $x=0$ and $x=110$. This is due to the fact that the particle centroid cannot sample the no-slip condition. Also note the blunting of the suspension velocity profile across the channel. This is also due to the finite size of the suspended species. Specifically, to get the velocity of an individual, non-interacting particle, one would have to integrate the impinging velocity profile over the surface of the entire particle, and we have parabolic flow, so the velocity profile is non-uniform. On the right panel, we show an interesting result/metric used to characterize suspension behavior in confined domains. More specifically, we refer to the suspension temperature. This is a temperature in the most fundamental sense, that is, we characterize the numbers of particle collisions with other surfaces across the channel. The majority of collisions occur where the fluid velocity gradient is steepest. In addition to exploring these suspension characteristics, we calculated a suspension viscosity to compare with the well-known Krieger correlation 


$$
\mu=\mu_{0}\left(1-\phi / \phi_{m}\right)^{-1.82}
$$

Here $\mu$ is the suspension viscosity, $\mu_{0}$ is the fluid viscosity, $\phi$ is the concentration of particles, and $\phi_{\mathrm{m}}$ is the concentration of particles at maximum packing. Our result and Eq. (8.1) were in excellent agreement. This is extremely useful to device designers, that is, it is paramount to know the suspension viscosity when deciding on pressure drops and volumetric flow rates.

\section{Electro-osmotic flow}

Finally, we adapted the LB method to handle electro-osmotic flows. This was accomplished by incorporating Eq. (6.1) in to the LB formulation at charged surfaces. The results are shown below in Figure 28.

EO Flow (2D Channel)

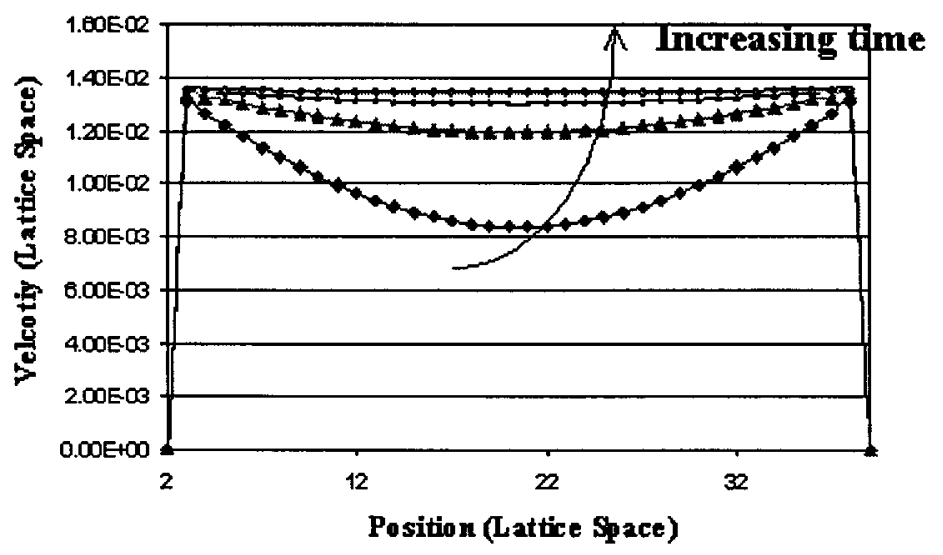

Figure 28 The velocity profile produced by Electro-Osmotic boundary condisions in a rectangular channel. The boundary condition at the channel wall was set by the Smolucalski equation for thin double layers.

This simple example demonstrates the capability as applied to a thin double layer configuration in a straight channel. Given the flexibility of the LB method, this result can easily be extended to more complex geometries, variable Zeta potential and include suspended species. However, during the Program, we were asked to target our efforts specifically to what our customers needed. Our customer base was predominately associated with Dielectrophoresis. Only in the last few months did we identify a customer who needed our capability to study suspension mechanics in EO flow; unfortunately, we had all of our resources focused on DEP at the time. As a result, this work was not performed by our team. 


\section{Gantt}

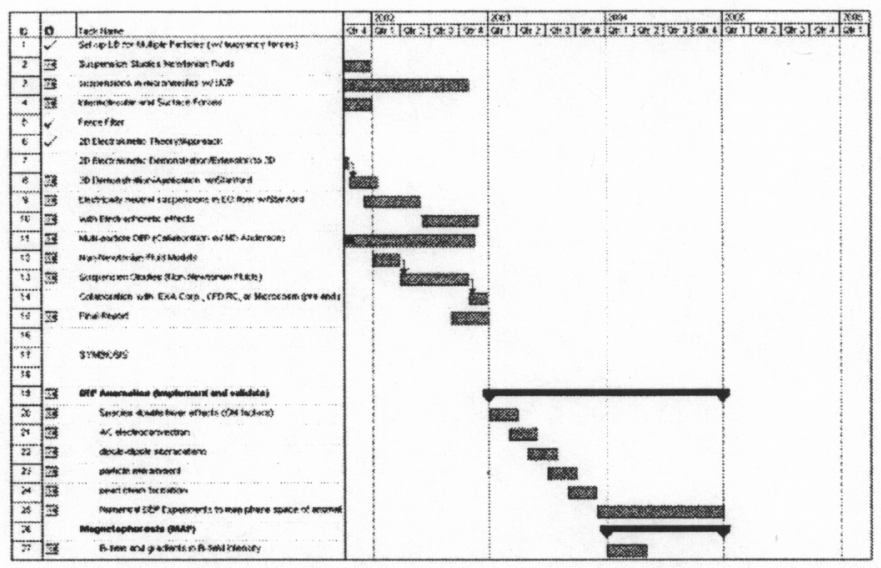

Figure 29 Gantt Chart for the project aims as put forward in the original propsal.

\section{Conclusions}

The lattice Boltzmann method is an extremely useful approach for studying particle dynamics in microflows. The approach readily permits the inclusion of new physics. The limitations include the following: 1) the computational domain is limited to sub-systems. As a result, inlet and outlet boundary conditions need to be specified using theory or another numerical approach; and 2) the approach is limited to $\mathrm{mm}$ length scales and micro-second time scales; therefore it is an improvement over MD simulations, but to study systems of interest, the approach must be made parallel and requires significant computational facilities, e.g., for large problems. In spite of these limitations, the PI and team found that the LB method is useful, and we are continuing to develop this capability to cover additional application space. Additionally, all of the physics modules developed through this program are stand alone, are of great benefit and run on a single processor machine.

\section{Recommendations}

The lattice Boltzmann method is extremely useful, and therefore, I recommend continued support and use of this approach in the characterization of microflows. Given this foundation, I recommend that an effort to integrate the LB method to molecular level simulation and macroscale simulation be made. The resulting capability would be a very powerful computational tool that would link the effects of molecular level phenomena to macroscale performance. 
Papers and Presentations Resulting from this effort:

\section{Articles in refereed journals:}

D.S. Clague and E.K. Wheeler, "Dielectrophoretic manipulation of macromolecules: The Efield," Phys. Rev E., 64:26605 (2001).

D. Clague and P. Cornelius, "A numerical study of the hydrodynamic force and torque acting on a bounded spherical body in Poiseuille flow," Int. J. Numer. Meth in Fluids, 35:55 (2001).

M. Garcia and D.S. Clague, "The 2D electric field above a planar sequence of independent strip electrodes," J. of Phys. D-App Phys, 33: 2669 (2000).

\section{Articles in preparation:}

D.S. Clague and E.K. Wheeler, "Dielectrophoretic manipulation of macromolecules: Part 2 Dynamic simulation using the Lattice Boltzmann method."

D.S. Clague, E.K. Wheeler and P.R.C. Gascoyne, "Dielectrophoretic manipulation and the effects of the electric double layer."

T.H. Weisgraber, D. Trebotich and D.S. Clague "On the lattice Boltzmann method and fluid mechanics in complex microenvironments: a sudden contraction."

\section{Presentations:}

Clague, D.S. and E. K. Wheeler, "Dielectrophoretic Manipulation of Particles and the Influence of the Electric Double Layer, to be presented at the 2003 AIChE national meeting.

Clague, D.S. and E. K. Wheeler, "Dynamic Simulation of Dielectrophoretic Manipulation of Spherical Macromolecules in Microflows", 2001 AIChE national meeting.

Weisgraber, T H, Wheeler, E K and Clague D S, "Dynamic Simulation of Fluids and Suspensions in Microflows," 2001 AIChE national meeting.

Clague, D.S. and Wheeler, E.K. "Dynamic Simulation of Dielectrophoretic Manipulation of Spherical Macromolecules in Microflows." First Gordon Conference on the Chemistry and Physics of Microfluidics July 29-August 2, 2001.

Clague, D.S., "Lattice Boltzmann simulation of microfluidic devices", Presented at the Center for Computational Engineering Annual Review, Feb. 2001.

Clague, D.S., "Lattice Boltzmann simulation of microfluidic devices", Presented at the Center for Microtechnology Annual Review, Jan. 2001. 
Clague, Wheeler, Radford and Hon "Particulate Transport and Manipulation in Bounded Microflows," presented at the National AIChE conference in Los Angeles, '00'.

Clague "Lattice Boltzmann Simulation of Particle-Laden Flows in Microfluidic Devices," presented at the Center for Computational Engineering ' 01 ' review.

Clague "Lattice Boltzmann Simulation of Particle-Laden Flows in Microfluidic Devices," presented at the CMT ' 01 ' review.

Clague and Wheeler "Dynamic Simulation of Dielectrophoretic Manipulation of Spherical Macromolecules in Microflows," Gordon Conference on Microfluidics

July 29-August 2, 2001

Not included are all of the presentations and posters at DARPA PI meetings. Also, we did not include work that was not performed on this project, i.e., work performed prior to the program, work by other researchers performed before or during the program, or work performed on other contracts.

\section{Bibliography}

1. Chen, S. and G. Doolen, Lattice Boltzmann method for fluid flows. Annual Rev. Fluid Mechanics, 1998. 30: p. 329-364.

2. Ladd, A.J.C., Numerical simulation of particulate suspensions via a disretetized Boltzmann eqaution. Part 2. Numerical Results. J. Fluid Mech., 1994. 271: p. 311-339.

3. Ladd, A.J.C., Numerical simulation of particulate suspensions via a discretetized Boltzmann equation. Part 1. Theoretical Foundation. J. Fluid Mech., 1994. 271: p. 285309.

4. Israelachvili, J., Intermolecular and surface forces, ed. A. Press. 1992: Academic Press.

5. Anderson, J., Colloid transport by interfacial forces. Annual Rev. Fluid Mechanics, 1989. 21: p. 61.

6. Garcia, M. and D.S. Clague, The $2 D$ electric field above a planar sequence of independent strip electrodes. J. of Appl. Physics, 2000.

7. Jones, T.B., Electromechanics of Particles. 1995: Cambridge University Press.

8. Clague, D.S. and E.K. Wheeler, Dielectrophoretic manipulation of macromolecules: Part 1, The electric field. Physical Rev. E, 2001. 64(2).

9. Clague, D.S. and E.K. Wheeler, Dynamic simulation of dielectrophoretic manipulation of spherical macromolecules in microflows, in National AIChE conference. 2001: Reno, NV.

10. Clague, D.S.a.W., E.K, Dynamic Simulation of Dielectrophoretic Manipulation of Spherical Macromolecules in Microflows, in Gordon Confernece, the Chemistry and Physics of Microfluidics. 2001: Oxford England.

11. Morgan, $\mathrm{H}$., et al., The dielectrophoretic and travelling wave forces generated by interdigitated electrode arrays: analytical solution using Fourier series. J. Phys. D: Appl. Phys., 2001. 34: p. 1553-1561. 
12. Chen, S. and D. Martinez, On boundary conditions in lattice Boltzmann methods. Phys. Fluids, 1996. 8(9): p. 2527-2536.

13. Nott, P. and J.F. Brady, Pressure-driven flow of suspensions: simulation and theory. J. Fluid Mech., 1994. 275: p. 157.

This work was performed under the auspices of the U.S. Department of Energy by University of California, Lawrence Livermore National Laboratory under Contract W-7405-Eng-48. 